International Electronic Journal of Algebra

Volume 25 (2019) 129-170

DOI: $10.24330 /$ ieja.504139

\title{
ON LATTICES OF INTEGRAL GROUP ALGEBRAS AND SOLOMON ZETA FUNCTIONS
}

\author{
Susanne Danz and Tommy Hofmann \\ Received: 14 May 2018; Accepted: 17 October 2018 \\ Communicated by Burkhard Külshammer
}

\begin{abstract}
We investigate integral forms of certain simple modules over group algebras in characteristic 0 whose $p$-modular reductions have precisely three composition factors. As a consequence we, in particular, complete the description of the integral forms of the simple $\mathbb{Q S}_{n}$-module labelled by the hook partition $\left(n-2,1^{2}\right)$. Moreover, we investigate the integral forms of the Steinberg module of finite special linear groups $\operatorname{PSL}_{2}(q)$ over suitable fields of characteristic 0 . In the second part of the paper we explicitly determine the Solomon zeta functions of various families of modules and lattices over group algebra, including Specht modules of symmetric groups labelled by hook partitions and the Steinberg module of $\operatorname{PSL}_{2}(q)$.
\end{abstract}

Mathematics Subject Classification (2010): 20C10, 20C11, 20C30, 20C20, $11 \mathrm{~S} 45$

Keywords: Integral representation, lattice, Jordan-Zassenhaus, symmetric group, Specht module, hook partition, projective special linear group, Steinberg module, Solomon zeta function

\section{Introduction}

In this paper we continue our study of integral representations of symmetric groups, begun in [5]. Let $\mathfrak{S}_{n}$ be the symmetric group of degree $n \geqslant 0$. Moreover, for every partition $\lambda$ of $n$, let $S_{\mathbb{Q}}^{\lambda}$ be the corresponding Specht $\mathbb{Q S}_{n}$-module. As $\lambda$ varies over the set of partitions of $n$, the Specht modules $S_{\mathbb{Q}}^{\lambda}$ yield representatives of the isomorphism classes of (absolutely) simple $\mathbb{Q S}_{n}$-modules. Every Specht module $S_{\mathbb{Q}}^{\lambda}$ is already equipped with a particular integral form, the Specht $\mathbb{Z}_{\mathfrak{S}_{n}}$-lattice $S_{\mathbb{Z}}^{\lambda}$. In light of the celebrated Jordan-Zassenhaus Theorem, it is, therefore, natural to ask for a description of all isomorphism classes of $\mathbb{Z} \mathfrak{S}_{n}$-lattices that are $\mathbb{Z}$-forms of a given Specht module $S_{\mathbb{Q}}^{\lambda}$, or at least for the number of these. To do so, a possible strategy is to consider, for each prime $p$, the $p$-adic completion $S_{\mathbb{Q}_{p}}^{\lambda}:=\mathbb{Q}_{p} \otimes_{\mathbb{Q}} S_{\mathbb{Q}}^{\lambda}$ and determine the $\mathbb{Z}_{p} \mathfrak{S}_{n}$-lattices that are $\mathbb{Z}_{p}$-forms of $S_{\mathbb{Q}_{p}}^{\lambda}$. In general, this is way too difficult a task. 
In [5] we investigated the case where $\lambda$ is a hook partition of $n \geqslant 3$, that is, a partition of the form $\left(n-r, 1^{r}\right)$, for some $r \in\{1, \ldots, n-2\}$. Specht modules (and Specht lattices) labelled by hook partitions have been studied a lot and much is known about their structure. Nevertheless, as far as the determination of the $\mathbb{Z}$-forms of $S_{\mathbb{Q}}^{\left(n-r, 1^{r}\right)}$ as concerned, we so far only have complete information in the case where $r=1$ : By work of Plesken [13] and Craig [3], the number of isomorphism classes of $\mathbb{Z}$-forms of $S_{\mathbb{Q}}^{(n-1,1)}$ equals the number of positive divisors of $n$, and one can give explicit representatives.

So, one may focus on the case where $r>1$. If $p$ is an odd prime, then the $\mathbb{Q}_{p} \mathfrak{S}_{n}$-module $S_{\mathbb{Q}_{p}}^{\left(n-r, 1^{r}\right)}$ admits precisely $\nu_{p}(n)+1$ isomorphism classes of $\mathbb{Z}_{p}$-forms, where $\nu_{p}(n)$ denotes the $p$-adic valuation of $n$. Explicit representatives of these isomorphism classes have been determined in [5, Theorem 6.1]; see also the work of Plesken in [14, Satz (III.8)] and [15, Theorem (VI.2)], who studied these modules using different methods.

The case where $p=2$ turned out to be considerably more difficult. In [5, Section 7], we were only able to give explicit representatives of the isomorphism classes of $\mathbb{Z}_{2}$-forms of the $\mathbb{Q}_{2} \mathfrak{S}_{n}$-module $S_{\mathbb{Q}_{2}}^{\left(n-2,1^{2}\right)}$, and only if $n \neq \equiv(\bmod 4)$. One aim of the present paper is to settle the remaining case $n \equiv 0(\bmod 4)$. This will be achieved in Theorem 3.7, which then entails Corollary 3.9 on the number of isomorphism classes of $\mathbb{Z}$-forms of $S_{\mathbb{Q}}^{\left(n-2,1^{2}\right)}$.

In fact, Theorem 3.7 will turn out to be a special instance of the more general result in Theorem 3.2. The latter deals with the following situation: suppose that $G$ is a finite group, $R$ is a principal ideal domain with field of fractions $K$ of characteristic 0 and residue field $k:=R / \mathbf{J}(R)$ of characteristic $p>0$. Suppose further that $V$ is an absolutely simple $K G$-module with an $R$-form $L$ whose modular reduction $k \otimes_{R} L$ has, as $k G$-module, precisely three composition factors satisfying some additional properties. Then we shall determine all $R$-forms of the $K G$-module $V$ up to isomorphism.

The hypotheses of Theorem 3.2 might at first seem rather special. In Proposition 3.18, we shall see a second application of this result to the case where $G$ is a finite projective special linear group of degree 2 and $V$ is the Steinberg module of $K G$, for suitable fields $K$ of characteristic 0 .

In light of this, we are tempted to ask whether Theorem 3.2 can be used to treat further finite groups and simple $K G$-modules arising as augmentation kernels of two-transitive permutation representations; see Question 3.19. At the moment we are, however, not able to answer this question. 
In Section 4 we then investigate the (Solomon) zeta functions of various families of $\mathbb{Z} G$-lattices, where $G$ is a finite group. In [16] L. Solomon introduced a generalization of the Riemann zeta function with the aim to study enumerative problems in integral representation theory. Subsequently, Bushnell and Reiner intensively studied Solomon's zeta functions; see [2] for an overview of their theory.

In Section 4, we shall give a concise summary of Solomon's definitions and the properties of the Solomon zeta functions relevant to our applications. In the case where $G$ is a finite group and $L$ is a $\mathbb{Z} G$-lattice, the zeta function of $L$ is defined as

$$
\zeta_{\mathbb{Z} G}(L, s):=\sum_{N \subseteq L}[L: N]^{-s} \quad(s \in \mathbb{C}),
$$

where $N$ varies over all $\mathbb{Z} G$-sublattices of $L$ of finite index. This will usually be viewed as a formal Dirichlet series, disregarding questions of convergence.

The concrete computation of zeta functions of $\mathbb{Z} G$-lattices is in general a rather difficult problem, and not too much is known in this direction. The case where $L$ is the regular $\mathbb{Z} G$-lattice has been studied most intensively; for a list of known results see [6]. In [6], the second author determined the zeta functions $\zeta_{\mathbb{Z} \mathfrak{S}_{n}}(L, s)$, where $L$ is a $\mathbb{Z}$-form of the Specht $\mathbb{Q S}_{n}$-module labelled by the hook partition $\left(2,1^{n-2}\right)$. In Section 4.5 of the present paper we shall generalize the results of [6], and determine global and local zeta functions of further Specht lattices labelled by hook partitions. As well, in Section 4.6 we again consider the projective special linear group $\operatorname{PSL}_{2}(q)$, where $q$ is a prime power, and the Steinberg module of $\mathbb{Q}\left[\mathrm{PSL}_{2}(q)\right]$. We shall determine the zeta function of a distinguished $\mathbb{Z}$-form of this module. The key ingredient here will again be Theorem 3.2.

The present paper is organized as follows: In Section 2 we briefly summarize some properties of graduated orders that will be relevant in subsequent sections. Section 3 is then devoted to establishing Theorem 3.2 and its applications to the study of integral forms of the Specht $\mathbb{Q S}_{n}$-module $S_{\mathbb{Q}}^{\left(n-2,1^{2}\right)}$ and the Steinberg module of $\mathbb{Q}\left[\mathrm{PSL}_{2}(q)\right]$, respectively. In Section 4 we recall Solomon's notion of global and local zeta functions of modules over group algebra. We then explicitly compute these zeta functions for various families of modules and lattices, including Specht modules of symmetric groups labelled by hook partitions, and the Steinberg module of $\operatorname{PSL}_{2}(q)$.

\section{Notation and prerequisites}

In this section we fix some notation, briefly recall the notion of a graduated order, and summarize the known results that will be relevant in Section 3 later. We 
follow the work of Plesken on the subject, and refer the reader to [14] and [15] for further background.

Notation 2.1. (a) Let $F$ be any field, and let $A$ be a finite-dimensional $F$-algebra. An $A$-module is always supposed to be a finitely generated left module. For an $A$ module $V$, we denote by $\operatorname{Rad}(V)$ the Jacobson radical of $V$, and by $\operatorname{Hd}(V):=$ $V / \operatorname{Rad}(V)$ the head of $V$. The socle of $V$ will be denoted by $\operatorname{Soc}(V)$.

(b) Let $R$ be a principal ideal domain with field of fractions $K$, let $\mathfrak{m}=(\pi)$ be a maximal ideal in $R$, and let $k:=R / \mathfrak{m}$ be the corresponding residue field. By an $R$-order we understand a finitely generated $R$-algebra $\Lambda$ that is free over $R$ of finite $R$-rank. One has a $k$-algebra isomorphism $k \otimes_{R} \Lambda \cong \Lambda / \mathfrak{m} \Lambda$; for convenience, we shall often identify these algebras and denote them simply by $k \Lambda$. A $\Lambda$-lattice is then a finitely generated left $\Lambda$-module $L$ that is $R$-free of finite $R$-rank, which we denote by $\operatorname{rk}_{R}(L)$. The factor module $\bar{L}:=L / \mathfrak{m} L$ naturally carries the structure of a $k \Lambda$-module, and $L / \mathfrak{m} L \cong k \otimes_{R} L$.

If $A$ is a finite-dimensional $K$-algebra and if an $R$-order $\Lambda$ is a subring of $A$ with $K \Lambda=A$, then one calls $\Lambda$ an $R$-order in $A$. In this case, we also identify the $K$-algebras $A$ and $K \otimes_{R} \Lambda$. If $V$ is an $A$-module and $L$ is a $\Lambda$-lattice such that $K \otimes_{R} L \cong V$ as $A$-modules, then one calls $L$ an $R$-form of $V$. As usual, we shall often work with an $R$-form $L$ of $V$ such that $L \subseteq V$. Moreover, recall that every $R$-form of $V$ is isomorphic to a $\Lambda$-sublattice of any given $R$-form $L$.

(c) With the notation as in (b), suppose that $L$ is a $\Lambda$-lattice, and let $L^{\prime} \subseteq L$ be a $\Lambda$-sublattice of $L$ with $\operatorname{rk}_{R}(L)=\operatorname{rk}_{R}\left(L^{\prime}\right)$. If $L^{\prime} \subseteq \pi^{i} L$, for some $i \in \mathbb{N}$, then we denote by $L^{\prime} / \pi^{i}$ the $\Lambda$-sublattice $\left\{\pi^{-i} x: x \in L^{\prime}\right\}$ of $L$, which satisfies $\pi^{i}\left(L^{\prime} / \pi^{i}\right)=L^{\prime}$ and is isomorphic to $L^{\prime}$.

(d) Now suppose that $R$ is local, and again let $\Lambda$ be an $R$-order. We shall call a $\Lambda$-module $L$ simple if $L \neq\{0\}$ and if $L$ and $\{0\}$ are the only $\Lambda$-submodules of $L$. If $L$ is a simple $\Lambda$-module, then $\mathfrak{m} L=\pi L$ is a $\Lambda$-submodule of $L$, and $\mathfrak{m} L \neq L$, by Nakayama's Lemma $[4,(30.2)]$. Thus $\mathfrak{m} L=\{0\}$; in particular, every simple $\Lambda$-module is a torsion module. This shows that $\mathfrak{m} \Lambda$ is contained in the Jacobson radical $\mathbf{J}(\Lambda)$ of $\Lambda$, which entails $k$-algebra isomorphisms

$$
\Lambda / \mathbf{J}(\Lambda) \cong(\Lambda / \mathfrak{m} \Lambda) /(\mathbf{J}(\Lambda) / \mathfrak{m} \Lambda)=(\Lambda / \mathfrak{m} \Lambda) / \mathbf{J}(\Lambda / \mathfrak{m} \Lambda) \cong k \Lambda / \mathbf{J}(k \Lambda) ;
$$

in particular, there are bijections between the isomorphism classes of simple modules of $\Lambda, \Lambda / \mathbf{J}(\Lambda), k \Lambda$ and $k \Lambda / \mathbf{J}(k \Lambda)$, respectively. 
If $L$ is any (finitely generated) $\Lambda$-module, then one also has $\pi L=\mathfrak{m} L=\mathfrak{m} \Lambda \cdot L \subseteq$ $\mathbf{J}(\Lambda) \cdot L \subseteq \operatorname{Rad}(L)$, and

$$
L / \operatorname{Rad}(L) \cong(L / \mathfrak{m} L) /(\operatorname{Rad}(L) / \mathfrak{m} L)=(L / \mathfrak{m} L) /(\operatorname{Rad}(L / \mathfrak{m} L)),
$$

as $\Lambda$-modules and $k \Lambda$-modules.

For simplicity, for the remainder of this section, $R$ will be a local principal ideal domain with maximal ideal $\mathfrak{m}=(\pi)$, field of fractions $K$ and residue field $k:=R / \mathfrak{m}$.

Definition 2.2. Let $n \in \mathbb{N}$. An $R$-order $\Lambda$ in the matrix algebra $A:=K^{n \times n}$ is called a graduated $R$-order in $A$ if there exist pairwise orthogonal idempotents $e_{1}, \ldots, e_{n} \in \Lambda$ such that $1_{A}=1_{\Lambda}=e_{1}+\cdots+e_{n}$.

Remark 2.3. Suppose that $A$ is any semisimple $K$-algebra, and let $\varepsilon$ be a block idempotent of $A$, that is, a projection onto one of the Wedderburn components of $A$. If $K$ is a splitting field of the simple $K$-algebra $\varepsilon A \varepsilon$, then $\varepsilon A \varepsilon \cong K^{n \times n}$, where $n$ is the dimension of the (up to isomorphism uniquely determined) simple $\varepsilon A \varepsilon$-module. This is the situation we shall investigate in the following.

2.4. Exponent matrices and normal form. (a) Following [14, Definition (I.3)] and [15, Definition (II.1), (II.2)], consider $r, n, d_{1}, \ldots, d_{r} \in \mathbb{N}$ with $n=d_{1}+\cdots+d_{r}$ as well as a matrix $M=\left(m_{i j}\right) \in \mathbb{Z}^{r \times r}$ all of whose entries are non-negative. Then the set of block matrices

$$
\Lambda:=\Lambda\left(d_{1}, \ldots, d_{r} ; M\right):=\left\{\left(a_{i j}\right) \in R^{n \times n}: a_{i j} \in \mathfrak{m}^{m_{i j}} \cdot R^{d_{i} \times d_{j}}\right\} \subseteq R^{n \times n} \subseteq K^{n \times n}
$$

is a (graduated) order in $K^{n \times n}$ if and only if, for all $i, j, k \in\{1, \ldots, r\}$, one has

$$
m_{i i}=0
$$

and

$$
m_{i j}+m_{j k} \geqslant m_{i k}
$$

If, moreover, one has

$$
m_{i j}+m_{j i}>0
$$

whenever $i \neq j$, one says that $\Lambda$ is in standard form, and calls $M$ the exponent matrix of $\Lambda$. By [15, Remark (II.3)], every graduated order in $K^{n \times n}$ is isomorphic to a graduated order in standard form.

Theorem 2.5 ([14, Satz (I.26)],[15, Theorem (II.16)]). Suppose that $A$ is a semisimple $K$-algebra, let $\Gamma$ be any $R$-order in $A$, and let $\varepsilon$ be a block idempotent of $A$. Moreover, let $V$ be an absolutely simple $A$-module with $\varepsilon V=V$, and let $L \subseteq V$ be 
a $\Gamma$-lattice that is an $R$-form of $V$. Then $\varepsilon \Gamma \varepsilon$ is a graduated order in $\varepsilon A \varepsilon$ if and only if the following conditions are satisfied:

(i) every composition factor of the $k \Gamma$-module $L / \mathfrak{m} L$ occurs with multiplicity $1 ;$

(ii) every composition factor of the $k \Gamma$-module $L / \mathfrak{m} L$ is absolutely simple.

In the course of this paper we shall apply Theorem 2.5 in the case where $A=K G$ is the group algebra of a finite group $G$ over $K$, and $\Gamma$ is the $R$-order $R G$ in $A$. Therefore, we recall how to obtain a graduated order in standard form in $\varepsilon K G \varepsilon$ that is isomorphic to $\varepsilon R G \varepsilon$.

2.6. Sublattices and exponent matrices. We keep the notation of Theorem 2.5, and suppose that conditions (i) and (ii) are satisfied. Denote the $R$-order $\varepsilon \Gamma \varepsilon$ of $\varepsilon A \varepsilon$ by $\Lambda$. Let $D_{1}, \ldots, D_{r}$ be the pairwise non-isomorphic composition factors of the $k \Gamma$-module $L / \mathfrak{m} L$, with $k$-dimensions $d_{1}, \ldots, d_{r}$.

(a) As mentioned in 2.1, one has bijections between the isomorphism classes of simple modules of $\Lambda, \Lambda / \mathbf{J}(\Lambda), k \Lambda$ and $k \Lambda / \mathbf{J}(k \Lambda)$, respectively. Analogously, $\mathfrak{m} \Gamma \subseteq \mathbf{J}(\Gamma)$, and one has bijections between the isomorphism classes of simple modules of $\Gamma, \Gamma / \mathbf{J}(\Gamma), k \Gamma$ and $k \Gamma / \mathbf{J}(k \Gamma)$, respectively.

Since $L \subseteq V$ and $\varepsilon$ acts as the identity on $V$, it also acts as the identity on $L$ and all its sublattices. In particular, $L=\varepsilon L$ is also a $\Lambda$-lattice, and the $\Gamma$-sublattices of $L$ are just the inflations of the $\Lambda$-sublattices of $L$, along the surjective $R$-algebra homomorphism $\Gamma \rightarrow \Lambda=\varepsilon \Gamma \varepsilon, a \mapsto \varepsilon a$.

We may also view $L / \mathfrak{m} L$ both as $k \Gamma$ and $k \Lambda$-module. Since $V$ is, up to isomorphism, the only simple $\varepsilon A \varepsilon$-module and since $\Lambda$ is an $R$-order in $\varepsilon A \varepsilon$, the simple $k \Lambda$-modules arise precisely as the composition factors of the $k \Lambda$-module $L / \mathfrak{m} L$.

On the other hand, if $D$ is a simple $\Lambda$-module, then $D$ also becomes a simple $\Gamma$-module via inflation along the surjective $R$-algebra homomorphism $\Gamma \rightarrow \Lambda=$ $\varepsilon \Gamma \varepsilon, a \mapsto \varepsilon a$.

Thus, altogether, the simple $k \Gamma$-modules $D_{1}, \ldots, D_{r}$ may also be viewed as simple $k \Lambda$-modules. As such they are the composition factors of the $k \Lambda$-module $L / \mathfrak{m} L$. Moreover, $D_{1}, \ldots, D_{r}$ are representatives of the isomorphism classes of simple $k \Lambda$ modules.

(b) As observed in (a), every $\Gamma$-sublattice of $L$ is also a $\Lambda$-sublattice of $L$, and conversely. So consider the lattice of $\Gamma$-sublattices of $L$ of full $R$-rank. By [14, Folgerung (I.24)], [15, Remark (II.4); p. 14], for each $i \in\{1, \ldots, r\}$, there is a unique sublattice $L_{i}$ of $L$ such that $L_{i} \nsubseteq \mathfrak{m} L$ and $L_{i} / \operatorname{Rad}\left(L_{i}\right) \cong D_{i}$ as $k \Gamma$-modules. 
Then, for $i, j \in\{1, \ldots, r\}$, let $m_{i j} \in \mathbb{N}_{0}$ be the multiplicity of $D_{i}$ as a composition factor of $L / L_{j}$. Setting $M_{L}:=\left(m_{i j}\right)$, one deduces $\Lambda \cong \Lambda\left(d_{1}, \ldots, d_{r} ; M_{L}\right)$ as $R$ orders, and $\Lambda\left(d_{1}, \ldots, d_{r} ; M_{L}\right)$ is in standard form. The definition of $M_{L}$ of course depends on $L$ as well as on the ordering of the simple modules $D_{1}, \ldots, D_{r}$. However, once the latter ordering has been fixed and $L$ and $L^{\prime}$ are $R$-forms of $V$, [14, Satz (I.7)], [15, Proposition (II.6)] show that $M_{L}=M_{L^{\prime}}$ if and only of $L \cong L^{\prime}$ as $\Gamma$-lattices.

By [14, Satz (I.23)], [15, Remark (II.4)], every projective indecomposable $\Lambda$ module is isomorphic to a $\Lambda$-sublattice of $L$ of full rank. More precisely, if, for $i \in\{1, \ldots, r\}, P_{i}$ denotes a projective cover of the simple $\Lambda$-module $D_{i}$, then $P_{i} \cong L_{i}$ as $\Lambda$-modules. Note that, as $\Gamma$-module, $L_{i}$ is, in general, not projective.

(c) Again let $L \subseteq V$ be a $\Lambda$-lattice that is an $R$-form of $V$, and let $M_{L}=\left(m_{i j}\right)$ be the corresponding exponent matrix, so that $\Lambda \cong \Lambda\left(d_{1}, \ldots, d_{r} ; M_{L}\right)$. By [14, Satz (I.8)], [15, Remark (II.4)], there is a bijection between the set of isomorphism classes of $\Lambda$-lattices that are $R$-forms of $V$ and the set of $r$-tuples $\left(m_{1}, \ldots, m_{r}\right) \in \mathbb{N}_{0}^{r}$ satisfying

$$
m_{i j}+m_{j} \geqslant m_{i}, \text { for } i, j \in\{1, \ldots, r\}
$$

and

$$
m_{k}=0, \text { for some } k \in\{1, \ldots, r\} \text {. }
$$

More precisely, to each such $r$-tuple $\left(m_{1}, \ldots, m_{r}\right)$, one associates the unique fullrank $\Lambda$-sublattice $L\left(m_{1}, \ldots, m_{r}\right)$ of $L$ such that, for $i \in\{1, \ldots, r\}$, the simple $\Lambda$ module $D_{i}$ occurs with multiplicity $m_{i}$ as a composition factor of $L / L\left(m_{1}, \ldots, m_{r}\right)$.

(d) Now suppose that $A=K G$ and $\Gamma=R G$, for a finite group $G$. Then $k \Gamma \cong k G$. Consider the simple $A$-module $V^{*}$, that is, the $K$-linear dual of $V$, let $\varepsilon^{*}$ be the block idempotent of $A$ with $\varepsilon^{*} V^{*}=V^{*}$, and let $T \subseteq V^{*}$ be an $R$-form of $V^{*}$. Then $V^{*}$ and $T$ also satisfy the hypotheses of Theorem 2.5. The composition factors of the $k G$-module $T / \mathfrak{m} T$ are isomorphic to the simple $k G$-modules $D_{1}^{*}, \ldots, D_{r}^{*}$. If $V$ is a self-dual $K G$-module, and if $D_{1}, \ldots, D_{r}$ are self-dual $k G$-modules, then we may take $T \cong L$ and, by [14, Satz (III.1)] and [15, Proposition (IV.1)], we obtain

$$
m_{i j}+m_{j k}+m_{k i}=m_{j i}+m_{k j}+m_{i k}
$$

for $i, j \in\{1, \ldots, r\}$. It should be mentioned that, at the beginning of [14, Chapter III], $L$ is assumed to be projective, when viewed as $\varepsilon R G \varepsilon$-module. In our applications, we shall usually work with lattices that do not have this property. The assertion of [14, Satz(III.1)(i)] is, however, valid without any restrictions on $L$. 


\section{On simple $K G$-modules with three modular composition factors}

Throughout this section, let $R$ be a principal ideal domain with maximal ideal $\mathfrak{m}=(\pi)$, residue field $k$ of characteristic $p>0$, and field of fractions $K$ of characteristic 0 . Moreover, let $G$ be a finite group.

3.1. Submodule lattices. In this subsection, we shall investigate $R$-forms of particular absolutely simple $K G$-modules with three modular composition factors. Theorem 3.2 below will subsequently be applied to two examples on finite symmetric and projective special linear groups, respectively. Throughout this subsection suppose that $R$ is local.

Hypotheses 3.1. Let $V$ be an absolutely simple $K G$-module, and let $S_{1}:=L \subseteq V$ be an $R$-form of $V$ satisfying the following properties:

(a) there are pairwise non-isomorphic simple $k G$-modules $D_{1}, D_{2}$ and $D_{3}$ with $k$-dimensions $d_{1}, d_{2}$ and $d_{3}$, respectively, such that $d_{2} \neq d_{1} \neq d_{3}$ and $d_{1} \neq$ $d_{2}+d_{3}$, and such that the $k G$-module $S_{1} / \pi S_{1}$ has radical isomorphic to $D_{1}$ and head isomorphic to $D_{2} \oplus D_{3}$;

(b) $D_{1} \cong D_{1}^{*}$ and $\left(D_{2} \oplus D_{3}\right)^{*} \cong D_{2} \oplus D_{3}$;

(c) $\operatorname{Soc}\left(S_{1} / \pi S_{1}\right)=\operatorname{Rad}\left(S_{1} / \pi S_{1}\right)$;

(d) $S_{2}$ and $S_{3}$ are the maximal sublattices of $S_{1}$ with $S_{1} / S_{2} \cong D_{2}$ and $S_{1} / S_{3} \cong$ $D_{3}$ as $k G$-modules;

(e) there is some $t \in \mathbb{N}$ and, for each $i \in\{1, \ldots, t\}$, there is some sublattice $S_{3 i+1}$ of $S_{1}$ such that

(i) for each $i \in\{0, \ldots, t-1\}, \pi S_{3 i+1} \subseteq S_{3(i+1)+1} \subseteq S_{3 i+1}$ and the dimension satisfies $\operatorname{dim}_{k}\left(S_{3(i+1)+1} / \pi S_{3 i+1}\right)=d_{1}$;

(ii) $\forall i \in\{1, \ldots, t-1\}: S_{3 i+1} / \pi S_{3 i+1} \cong D_{1} \oplus D_{2} \oplus D_{3}$;

(iii) $S_{3 t+1} \cong S_{1}^{*}$.

Theorem 3.2. Suppose that Hypotheses 3.1 hold. Then one has the following:

(a) $S_{4}$ is the unique common maximal sublattice of $S_{2}$ and $S_{3}$; moreover, $S_{2} / S_{4} \cong$ $D_{3}$ and $S_{3} / S_{4} \cong D_{2}$;

(b) for $i \in\{1, \ldots, t-1\}$, the lattice $S_{3 i+1}$ has precisely three maximal sublattices $S_{3 i+2}, S_{3 i+3}$ and $\pi S_{3(i-1)+1}$, where $S_{3 i+1} / S_{3 i+2} \cong D_{2}, S_{3 i+1} / S_{3 i+3} \cong D_{3}$ and $S_{3 i+1} / \pi S_{3(i-1)+1} \cong D_{1}$;

(c) for $i \in\{1, \ldots, t-1\}$, the lattice $S_{3 i+2}$ has at least two maximal sublattices $S_{3(i+1)+1}$ and $\pi S_{3(i-1)+2}$; moreover, $S_{3 i+2} / S_{3(i+1)+1} \cong D_{3}$ and $S_{3 i+2} / p S_{3(i-1)+2} \cong$ $D_{1}$ 
(d) for $i \in\{1, \ldots, t-1\}$, the lattice $S_{3 i+3}$ has at least two maximal sublattices $S_{3(i+1)+1}$ and $\pi S_{3(i-1)+3}$; moreover, $S_{3 i+3} / S_{3(i+1)+1} \cong D_{2}$ and $S_{3 i+3} / \pi S_{3(i-1)+3} \cong$ $D_{1}$

(e) $\pi S_{3(t-1)+1}$ is the unique maximal sublattice of $S_{3 t+1}$, and $S_{3 t+1} / \pi S_{3(t-1)+1} \cong$ $D_{1}$

(f) the $R G$-lattices $S_{1}, \ldots, S_{3 t+1}$ are pairwise non-isomorphic $R$-forms of $V$. If $S_{4}$ is the unique maximal sublattice of $S_{2}$ as well as the unique maximal sublattice of $S_{3}$, then $S_{1}, \ldots, S_{3 t+1}$ are representatives of the isomorphism classes of $R$-forms of $V$, and $S_{1}$ has the following full-rank sublattices:

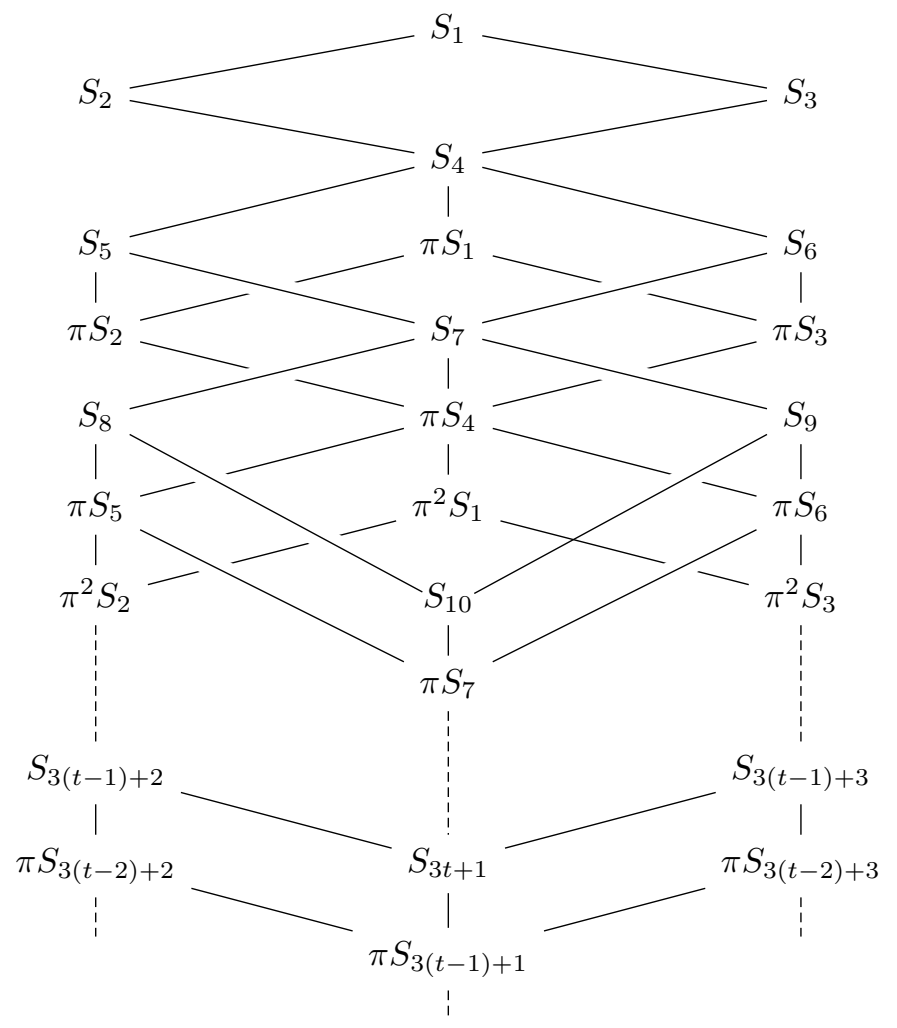

(g) if $D_{1}, D_{2}$ and $D_{3}$ are absolutely simple, let $\varepsilon$ be the block idempotent of $K G$ corresponding to $V$. Then $\Lambda:=\varepsilon R G \varepsilon$ is a graduated $R$-order in $A:=\varepsilon K G \varepsilon$. One 
has $\Lambda \cong \Lambda\left(d_{1}, d_{2}, d_{3} ; M_{L}\right)$, where

$$
M_{L}=\left(\begin{array}{lll}
0 & 0 & 0 \\
t & 0 & a \\
t & b & 0
\end{array}\right),
$$

for some $a, b \in \mathbb{N}$. One has $a=1=b$ if and only if $S_{4}$ is the unique maximal sublattice of $S_{2}$ and the unique maximal sublattice of $S_{3}$.

Proof. Assertion (a) is clear from our hypotheses. To prove assertions (b)-(d) we argue by induction on $i$.

Suppose first that $i=1$. The lattice $S_{4}$ has three maximal sublattices, since $S_{4} / \pi S_{4} \cong D_{1} \oplus D_{2} \oplus D_{3}$ as $k G$-module. By our hypotheses, $\pi S_{1} \subseteq S_{4} \subseteq S_{1}$ and $\operatorname{dim}_{k}\left(S_{4} / \pi S_{1}\right)=d_{1}$. By Hypotheses 3.1(a), this forces $S_{4} / \pi S_{1} \cong D_{1}$; in particular, $\pi S_{1}$ is maximal in $S_{4}$. Now let $S_{5}$ and $S_{6}$ be the maximal sublattices of $S_{4}$ such that $S_{4} / S_{5} \cong D_{2}$ and $S_{4} / S_{6} \cong D_{3}$. Then we have $S_{5} / \pi S_{4} \cong D_{1} \oplus D_{3}$ and $S_{6} / \pi S_{4} \cong D_{1} \oplus D_{2}$. Moreover, from $\pi S_{4} \subseteq S_{7} \subseteq S_{4}$ and $\operatorname{dim}_{k}\left(S_{7} / \pi S_{7}\right)=d_{1}$ we deduce that $S_{7} / \pi S_{4} \cong D_{1}$, so that $S_{7} / \pi S_{4}$ is the unique submodule of $S_{4} / \pi S_{4}$ isomorphic to $D_{1}$. In particular, $S_{7} / \pi S_{4} \subseteq S_{5} / \pi S_{4}$ and $S_{7} / \pi S_{4} \subseteq S_{6} / \pi S_{4}$. This implies $S_{7} \subseteq S_{5}, S_{7} \subseteq S_{6}, S_{5} / S_{7} \cong D_{3}$, and $S_{6} / S_{7} \cong D_{2}$. Hence $S_{7}$ is maximal in both $S_{5}$ and $S_{6}$.

Next we show that $\pi S_{2}$ is maximal in $S_{5}, \pi S_{3}$ is maximal in $S_{6}$ and $S_{5} / \pi S_{2} \cong$ $D_{1} \cong S_{6} / \pi S_{3}$. First assume that $\pi S_{2} \nsubseteq S_{5}$. Since $\pi S_{2} \subseteq \pi S_{1} \subseteq S_{4}$ and since $S_{5}$ is maximal in $S_{4}$, this gives $S_{4}=S_{5}+\pi S_{2}=S_{5}+\pi S_{1}$ and

$$
S_{4} / \pi S_{4}=\left(S_{5} / \pi S_{4}\right)+\left(\pi S_{2} / \pi S_{4}\right) \cong\left(D_{1} \oplus D_{3}\right)+D_{3} .
$$

So either $\pi S_{2} / \pi S_{4} \subseteq S_{5} / \pi S_{4}$ or $S_{4} / \pi S_{4}=S_{5} / \pi S_{4} \oplus \pi S_{2} / \pi S_{4}$. Since we are assuming $\pi S_{2} \nsubseteq \nsubseteq S_{5}$, the first case cannot occur, implying $S_{4} / \pi S_{4} \cong D_{1} \oplus D_{3} \oplus D_{3}$, a contradiction, since $D_{2} \neq D_{3}$. Consequently, $\pi S_{2} \subseteq S_{5}$ and

$$
S_{5} / \pi S_{2} \cong\left(S_{5} / \pi S_{4}\right) /\left(\pi S_{2} / \pi S_{4}\right) \cong\left(D_{1} \oplus D_{3}\right) / D_{3} \cong D_{1}
$$

in particular, $\pi S_{2}$ is maximal in $S_{5}$ and $\pi S_{2} \neq S_{7}$.

Analogously, we deduce that $\pi S_{3} \neq S_{7}$ is maximal in $S_{6}$ with $S_{6} / \pi S_{3} \cong D_{2}$.

So suppose now that $i>1$. As above, we deduce that $S_{3 i+1}$ has precisely three maximal sublattices $\pi S_{3(i-1)+1}, S_{3 i+2}$ and $S_{3 i+3}$, where $S_{3 i+1} / \pi S_{3(i-1)+1} \cong D_{1}$, $S_{3 i+1} / S_{3 i+2} \cong D_{2}$ and $S_{3 i+1} / S_{3 i+3} \cong D_{3}$. Moreover, $S_{3 i+2}$ has at least two maximal sublattices $\pi S_{3(i-1)+2}$ and $S_{3(i+1)+1}$, with $S_{3 i+2} / \pi S_{3(i-1)+2} \cong D_{1}$ and $S_{3 i+2} / S_{3(i+1)+1} \cong D_{3}$. To see this, note that $S_{3(i+1)+1} / \pi S_{3 i+1}$ is the unique submodule of $S_{3 i+1} / \pi S_{3 i+1}$ isomorphic to $D_{1}$. Since $S_{3 i+1} / S_{3 i+2} \cong D_{2}$, we have 
$S_{3 i+2} / \pi S_{3 i+1} \cong D_{1} \oplus D_{3}$, implying $S_{3(i+1)+1} / \pi S_{3 i+1} \subseteq S_{3 i+2} / \pi S_{3 i+1}, S_{3(i+1)+1} \subseteq$ $S_{3 i+2}$ and $S_{3 i+2} / S_{3(i+1)+1} \cong D_{3}$. Assuming that $\pi S_{3(i-1)+2} \nsubseteq S_{3 i+2}$ and using that $S_{3(i-1)+2} / S_{3 i+1} \cong D_{3}$ by induction, we again obtain the contradiction

$D_{1} \oplus D_{2} \oplus D_{3} \cong S_{3 i+1} / \pi S_{3 i+1} \cong S_{3 i+2} / \pi S_{3 i+1} \oplus \pi S_{3(i-1)+2} / \pi S_{3 i+1} \cong D_{1} \oplus D_{3} \oplus D_{3}$

Hence $\pi S_{3(i-1)+2} \subseteq S_{3 i+2}$ and

$S_{3 i+2} / \pi S_{3(i-1)+2} \cong\left(S_{3 i+2} / \pi S_{3 i+1}\right) /\left(\pi S_{3(i-2)+1} / \pi S_{3 i+1}\right) \cong\left(D_{1} \oplus D_{3}\right) / D_{3} \cong D_{1}$.

This proves assertion (c), and assertion (d) concerning the maximal sublattices of $S_{3 i+3}$ is proved analogously.

To show (e), recall that $\operatorname{Soc}\left(S_{1} / \pi S_{1}\right) \cong D_{1}$ and $D_{1} \cong D_{1}^{*}$, by our hypotheses. Since $S_{3 t+1} \cong S_{1}^{*}$, we also have $S_{3 t+1} / \pi S_{3 t+1} \cong\left(S_{1} / \pi S_{1}\right)^{*}$, so that $S_{3 t+1} / \pi S_{3 t+1}$ has head isomorphic to $D_{1}$; in particular, $S_{3 t+1}$ has a unique maximal sublattice. By our hypotheses, we further know that $\pi S_{3(t-1)+1} \subseteq S_{3 t+1} \subseteq S_{3(t-1)+1}$ and $\operatorname{dim}_{k}\left(S_{3 t+1} / \pi S_{3(t-1)+1}\right)=\operatorname{dim}_{k}\left(D_{1}\right)$. Hence $S_{3 t+1} / \pi S_{3(t-1)+1} \cong D_{1}$, by Hypotheses 3.1(a), and $\pi S_{3(t-1)+1}$ is indeed the unique maximal sublattice of $S_{3 t+1}$.

To complete the proof of the theorem, it remains to settle (f) and (g). To do so we shall apply [13, Proposition 2.3]. We first note that neither of the lattices $S_{1}, \ldots, S_{3 t+1}$ is contained in $\pi S_{1}$. Namely, by Hypotheses 3.1(d) and assertions (a)(d) above, for $j \in\{1, \ldots, 3 t+1\}$, every composition factor of $S_{1} / S_{j}$ is isomorphic to $D_{2}$ or $D_{3}$, while $S_{1} / \pi S_{1}$ has a composition factor isomorphic to $D_{1}$. Hence, by [13, Proposition 2.3], the $R$-forms $S_{1}, \ldots, S_{3 t+1}$ are pairwise non-isomorphic $R G$-lattices. By construction, (7) is part of the submodule lattice of $S_{1}$.

Now suppose that both $S_{2}$ and $S_{3}$ have a unique maximal sublattice, which then has to be equal to $S_{4}$. We again argue by induction on $i$ to show that each of $S_{3 i+2}$ and $S_{3 i+3}$ has precisely two maximal sublattices, for all $i \in\{1, \ldots, t-1\}$. So let $i=1$, and assume that $S_{5}$ has a maximal sublattice $T$ with $S_{7} \neq T \neq$ $\pi S_{2}$. Then we must have $S_{5} / \pi S_{5} \cong D_{1} \oplus D_{2} \oplus D_{3}$, and $S_{5} / T \cong D_{2}$. Since $\pi S_{2} / \pi S_{5} \cong\left(\pi S_{2} / \pi^{2} S_{2}\right) /\left(\pi S_{5} / \pi^{2} S_{2}\right)$ and $\pi S_{5} / \pi^{2} S_{2} \cong S_{5} / \pi S_{2} \cong D_{1}$, we conclude that $\pi S_{2} / \pi S_{5} \cong D_{2} \oplus D_{3}$ is isomorphic to a factor module of $\pi S_{2} / \pi^{2} S_{2} \cong S_{2} / \pi S_{2}$. But $S_{2} / \pi S_{2}$ has a simple head isomorphic to $D_{3}$, hence does not have a factor module isomorphic to $D_{2} \oplus D_{3}$.

This proves the assertions concerning $S_{5}$, and the lattice $S_{6}$ is treated analogously.

Now let $i>1$. Assume that we have a maximal sublattice $T$ of $S_{3 i+2}$ with $\pi S_{3(i-1)+2} \neq T \neq S_{3(i+1)+1}$. Then, as in the case where $i=1$, we get $S_{3 i+2} / \pi S_{3 i+2} \cong$ 
$D_{1} \oplus D_{2} \oplus D_{3}$ and $S_{3 i+2} / T \cong D_{2}$. We have $\pi S_{3 i+2} \subseteq \pi S_{3 i+1} \subseteq \pi S_{3(i-1)+2}$, thus

$$
\pi S_{3(i-1)+2} / \pi S_{3 i+2} \cong\left(S_{3(i-1)+2} / \pi^{2} S_{3(i-1)+2}\right) /\left(\pi S_{3 i+2} / \pi^{2} S_{3(i-1)+2}\right)
$$

and

$$
\left(S_{3 i+2} / \pi S_{3 i+2}\right) /\left(\pi S_{3(i-1)+2} / \pi S_{3 i+2}\right) \cong S_{3 i+2} / \pi S_{3(i-1)+2} \cong D_{1} .
$$

Note that since we are assuming $S_{3 i+2} / \pi S_{3 i+2}$ to be semisimple, this implies that $\pi S_{3(i-1)+2} / \pi S_{3 i+2} \cong D_{2} \oplus D_{3}$. But we have just seen that $\pi S_{3(i-1)+2} / \pi S_{3 i+2}$ is isomorphic to a factor module of $\pi S_{3(i-1)+2} / \pi^{2} S_{3(i-1)+2} \cong S_{3(i-1)+2} / \pi S_{3(i-1)+2}$, which, by induction, has a head isomorphic to $D_{1} \oplus D_{3}$. Since $D_{1} \neq D_{2} \neq D_{3}$, we obtain a contradiction.

This proves the assertions concerning $S_{3 i+2}$, and the lattice $S_{3 i+3}$ is treated analogously. Consequently, we have now verified that (7) is the lattice of fullrank sublattices of $S_{1}$. Moreover, $S_{1}, \ldots, S_{3 t+1}$ are precisely those sublattices of $S_{1}$ of full rank that are not contained in $\pi S_{1}$, which are then representatives of the isomorphism classes of $R$-forms of $V$, by [13, Proposition 2.3]. This settles (f).

Lastly suppose that $D_{1}, D_{2}$ and $D_{3}$ are absolutely simple. Then $\Lambda$ is a graduated $R$-order in $A$, by Theorem 2.5. By (e), the lattice $S_{3 t+1}$ must be the unique sublattice of $S_{1}$ not contained in $\pi S_{1}$ that is a projective cover of $D_{1}$, when viewed as simple $\Lambda$-module as in 2.6(b). Consequently, $S_{1}$ is an injective $\Lambda$-lattice, and [14, Satz (I.23)(ii)], [15, Remark (II.4)] forces $m_{1 j}=0$, for $j \in\{1,2,3\}$.

Hence, by 2.6(b), with respect to the chosen ordering on $D_{1}, D_{2}, D_{3}$, we must have

$$
M_{L}=M_{S_{1}}=\left(\begin{array}{ccc}
0 & 0 & 0 \\
t & 0 & a \\
t & b & 0
\end{array}\right)
$$

for some $a, b \in \mathbb{N}$.

Consider the uniquely determined sublattices $P_{2}$ and $P_{3}$ of $S_{1}$ not contained in $\pi S_{1}$ such that $P_{2} / \operatorname{Rad}\left(P_{2}\right) \cong D_{2}$ and $P_{3} / \operatorname{Rad}\left(P_{3}\right) \cong D_{3}$. That is, $P_{2}$ is a projective cover of $D_{2}$ and $P_{3}$ is a projective cover of $D_{3}$, when viewed as $\Lambda$-modules. Then, by 2.6(b), we deduce that $S_{1} / P_{2}$ has only composition factors isomorphic to $D_{3}$, and the number of these is $b$. Similarly, $S_{1} / P_{3}$ has only composition factors isomorphic to $D_{2}$, and the number of these is $a$. Since $S_{2}$ and $S_{3}$ are the only maximal sublattices of $S_{1}$, this forces $P_{2} \subseteq S_{3}$ and $P_{3} \subseteq S_{2}$. Therefore, we have $a=b=1$ if and only if $P_{2}=S_{3}$ and $P_{3}=S_{2}$. This in turn is equivalent to $S_{2}$ and $S_{3}$ having a unique maximal sublattice, which then has to be the common sublattice $S_{4}$. 
Remark 3.3. Keep the notation of Theorem 3.2. Suppose that the simple $k G$ modules $D_{1}, D_{2}, D_{3}$ are absolutely simple and suppose also that $a=b=1$ in part (g). Then the $R G$-lattices $S_{1}, \ldots, S_{3 t+1}$ are representatives of the $R$-forms of $V$, and $\Lambda=\varepsilon R G \varepsilon$ is a graduated $R$-order of $\varepsilon K G \varepsilon$. Recall from 2.6(c) that the lattices $S_{1}, \ldots, S_{3 t+1}$ bijectively correspond to the triples $\left(m_{1}, m_{2}, m_{3}\right) \in \mathbb{N}_{0}^{3}$ satisfying the following conditions:

$$
\begin{aligned}
& t+m_{1} \geqslant m_{2} \geqslant m_{1} \\
& t+m_{1} \geqslant m_{3} \geqslant m_{1} \\
& 1+m_{3} \geqslant m_{2} \\
& 1+m_{2} \geqslant m_{3} .
\end{aligned}
$$

Since at least one of $m_{1}, m_{2}, m_{3}$ has to be 0 , this gives $m_{1}=0$ and

$$
\left(m_{1}, m_{2}, m_{3}\right) \in\{(0, j, j),(0, j+1, j),(0, j, j+1),(0, t, t): j \in\{0, \ldots, t-1\}\} .
$$

Moreover, by 2.6(c) and (7), the concrete correspondence between $S_{j}$ and the triple $\left(m_{1}, m_{2}, m_{3}\right)$ is given as follows:

\begin{tabular}{|c|c|}
\hline$j$ & $\left(m_{1}, m_{2}, m_{3}\right)$ \\
\hline \hline $3 j+1$ & $(0, j, j)$ \\
\hline $3 j+2$ & $(0, j+1, j)$ \\
\hline $3 j+3$ & $(0, j, j+1)$ \\
\hline $3 t+1$ & $(0, t, t)$ \\
\hline
\end{tabular}

where $j \in\{0, \ldots, t-1\}$.

3.2. Application I: symmetric groups. Our first application of Theorem 3.2 will be concerned with the symmetric group $\mathfrak{S}_{n}$ of degree $n \geqslant 0$. We begin by setting up some notation that will be chosen in accordance with [5]. For details on the representations of symmetric groups and the well-known properties of these used below, we refer the reader to [9].

Notation 3.4. Suppose that $R$ is a principal ideal domain with field of fractions $K$ of characteristic 0 . Moreover, let $(\pi)$ be a maximal ideal in $R$ such that the residue field $k:=R /(\pi)$ has characteristic $p>0$. The isomorphism classes of (absolutely) simple $K \mathfrak{S}_{n}$-modules are labelled by the partitions of $n$ : for every partition $\lambda$ of $n$, there is a simple $K \mathfrak{S}_{n}$-module $S_{K}^{\lambda}$, called the Specht $K \mathfrak{S}_{n}$-module labelled by $\lambda$, which carries a distinguished $R$-form $S_{R}^{\lambda}$, called the Specht $R \mathfrak{S}_{n}$-lattice labelled by $\lambda$. The $k \mathfrak{S}_{n}$-module $S_{R}^{\lambda} / \pi S_{R}^{\lambda}$ shall be denoted by $S_{k}^{\lambda}$. It is well known and easily 
deduced from the explicit construction of Specht modules in [8, Sections 4 and 8] that $S_{R}^{\lambda} \cong R \otimes_{\mathbb{Z}} S_{\mathbb{Z}}^{\lambda}$, for every partition $\lambda$ of $n$.

The isomorphism classes of (absolutely) simple $k \mathfrak{S}_{n}$-modules are labelled by the $p$-regular partitions of $n$, that is, partitions $\lambda$ of $n$ each of whose parts occurs with multiplicity at most $p-1$. The simple $k \mathfrak{S}_{n}$-module labelled by a $p$-regular partition $\mu$ is usually denoted by $D_{k}^{\mu}$. Recall also that $D_{k}^{\mu}$ is isomorphic to the head of $S_{k}^{\mu}$.

Every simple $K \mathfrak{S}_{n}$-module as well as every simple $k \mathfrak{S}_{n}$-module is self-dual.

In [5], we considered the case where $K \in\left\{\mathbb{Q}, \mathbb{Q}_{p}\right\}, R \in\left\{\mathbb{Z}, \mathbb{Z}_{p}\right\}$ and $k=\mathbb{F}_{p}$, for some prime number $p$. We studied the $R$-forms of the Specht $K \mathfrak{S}_{n}$-modules labelled by hook partitions $\left(n-r, 1^{r}\right)$, for $r \in\{0, \ldots, n-1\}$. In [5, Theorem 6.1] we determined a set of representatives of the isomorphism classes of $\mathbb{Z}_{p}$-forms of the Specht $\mathbb{Q}_{p} \mathfrak{S}_{n}$-module $S_{\mathbb{Q}_{p}}^{\left(n-r, 1^{r}\right)}$, for $p>2$ and $r \in\{0, \ldots, n-1\}$; see also [14, Satz (III.8)]. The main ingredient in the proof of our result were the results of Plesken [13] and Craig [3] on the case $r=1$. The case where $p=2$ turned out to be much more difficult. In [5, Theorem 7.10, Theorem 7.16] we settled the case $p=2=r$, for $n \not \equiv 0(\bmod 4)$. In Theorem 3.7 below we shall now treat the case $p=2=r$ and $n \equiv 0(\bmod 4)$, thereby verifying [5, Conjecture 7.18(a)]. For a summary of the known relationship between Specht modules labelled by hook partitions and exterior powers, we refer to $[5$, Section $4 ; 5.5]$.

In what follows, for every prime number $p$ and every $n \in \mathbb{N}$, we denote by $\nu_{p}(n)$ the $p$-adic valuation of $n$, that is, $\nu_{p}(n)=\max \left\{l \in \mathbb{N}_{0}: p^{l} \mid n\right\}$.

3.5. Specht modules labelled by hook partitions. (a) Suppose now that $p=2$ and $n \geqslant 4$. Let $t:=\nu_{2}(n)$. For $R \in\left\{\mathbb{Z}_{2}, \mathbb{Q}_{2}, \mathbb{F}_{2}\right\}$, one has $R \mathfrak{S}_{n}$-isomorphisms $S_{R}^{\left(n-2,1^{2}\right)} \cong \bigwedge^{2}\left(S_{R}^{(n-1,1)}\right)$; see [5, 5.5]. As in [5], we shall often view these isomorphisms as equalities, for convenience.

(b) Suppose that $p \mid n$. For $j \in\left\{0, \ldots, \nu_{2}(n)\right\}$, let $M_{2^{j}}$ be the $\mathbb{Z}_{2} \mathfrak{S}_{n^{-}}$-sublattice of $S_{\mathbb{Z}_{2}}^{(n-1,1)}$ in [5, Theorem 8.1]. Then

$$
S_{\mathbb{Z}_{2}}^{(n-1,1)}=M_{1} \supseteq M_{2} \supseteq M_{4} \supseteq \cdots \supseteq M_{2^{t}} \cong\left(S_{\mathbb{Z}_{2}}^{(n-1,1)}\right)^{*},
$$

and $M_{1}, \ldots, M_{2^{t}}$ are representatives of the isomorphism classes of $\mathbb{Z}_{2}$-forms of $S_{\mathbb{Q}_{2}}^{(n-1,1)}$. Moreover, for $j \in\{1, \ldots, t\}$, one has $2 M_{2^{j-1}} \subseteq M_{2^{j}} \subseteq M_{2^{j-1}},\left[M_{2^{j-1}}\right.$ : $\left.M_{2^{j}}\right]=2^{n-2}$ and $M_{2^{j-1}} / M_{2^{j}} \cong D_{\mathbb{F}_{2}}^{(n-1,1)}$ as $\mathbb{F}_{2} \mathfrak{S}_{n^{-}}$-modules. If $j \in\{1, \ldots, t-1\}$, then $M_{2^{j}} / 2 M_{2^{j}} \cong D_{\mathbb{F}_{2}}^{(n-1,1)} \oplus \mathbb{F}_{2}$.

By [5, Theorem 4.5], the $\mathbb{Z}_{2} \mathfrak{S}_{n}$-lattices $\bigwedge^{2}\left(M_{1}\right), \ldots, \bigwedge^{2}\left(M_{2^{t}}\right)$ are pairwise nonisomorphic $\mathbb{Z}_{2}$-forms of $S_{\mathbb{Q}_{2}}^{\left(n-2,1^{2}\right)}$. Lastly, recall that $S_{\mathbb{Z}_{2}}^{(n-1,1)}$ has rank $n-1$, and $D_{\mathbb{F}_{2}}^{(n-1,1)}$ has dimension $n-2$. 
Lemma 3.6. Let $n>4$ be such that $n \equiv 0(\bmod 4)$, and let $t:=\nu_{2}(n)$. Let $V$ be the absolutely simple $\mathbb{Q}_{2} \mathfrak{S}_{n}$-module $S_{\mathbb{Q}_{2}}^{\left(n-2,1^{2}\right)}$. Moreover, let $D_{1}:=D_{\mathbb{F}_{2}}^{(n-1,1)}$, $D_{2}:=D_{\mathbb{F}_{2}}^{(n)} \cong \mathbb{F}_{2}$, and $D_{3}:=D_{\mathbb{F}_{2}}^{(n-2,2)}$. With the notation as in 3.5(b), one has the following:

(a) $\bigwedge^{2}\left(M_{2^{i}}\right) \subseteq 2^{i} \bigwedge^{2}\left(M_{1}\right)=2^{i} S_{\mathbb{Z}_{2}}^{\left(n-2,1^{2}\right)}$, for $i \in\{0, \ldots, t\}$;

(b) for $i \in\{0, \ldots, t\}$, let $S_{3 i+1}:=\bigwedge^{2}\left(M_{2^{i}}\right) / 2^{i}$. Then $S_{1}, S_{4}, S_{7}, \ldots, S_{3 t+1}$ are $\mathbb{Z}_{2}$-forms of $V$ satisfying Hypotheses 3.1 .

Proof. As in the proof of [5, Proposition 6.5], we have $4 \bigwedge^{2}\left(M_{2^{i}}\right) \subseteq \bigwedge^{2}\left(M_{2^{i+1}}\right) \subseteq$ $2 \bigwedge^{2}\left(M_{2^{i}}\right)$, for $i \in\{0, \ldots, t-1\}$. Thus, by induction on $i$, we deduce that the modules $S_{1}, S_{4}, \ldots, S_{3 t+1}$ are $\mathbb{Z}_{2}$-forms of $V$ such that $2 S_{3 i+1} \subseteq S_{3(i+1)+1} \subseteq S_{3 i+1}$, for $i \in\{0, \ldots, t-1\}$.

Let $d_{i}:=\operatorname{dim}_{\mathbb{F}_{2}}\left(D_{i}\right)$, for $i \in\{1,2,3\}$. We have $S_{1} / p S_{1} \cong S_{\mathbb{F}_{2}}^{\left(n-2,1^{2}\right)}$, and it follows from [11, Theorem 1.1] that $\operatorname{Rad}\left(S_{\mathbb{F}_{2}}^{\left(n-2,1^{2}\right)}\right)=\operatorname{Soc}\left(S_{\mathbb{F}_{2}}^{\left(n-2,1^{2}\right)}\right) \cong D_{1}$ and $\operatorname{Hd}\left(S_{\mathbb{F}_{2}}^{\left(n-2,1^{2}\right)}\right) \cong D_{2} \oplus D_{3}$. Furthermore, $d_{1}=n-2$ and $d_{2}=1$. Thus $d_{3}=$ $\operatorname{dim}_{\mathbb{F}_{2}}\left(S_{\mathbb{F}_{2}}^{\left(n-2,1^{2}\right)}\right)-d_{1}-d_{2}=\left(\begin{array}{c}n-1 \\ 2\end{array}\right)-1-(n-2)$. From this one immediately deduces that Hypotheses 3.1(a) and (c) are satisfied. In particular, there is a unique maximal sublattice $S_{2}$ of $S_{1}$ with $S_{1} / S_{2} \cong D_{2}$, and a unique maximal sublattice $S_{3}$ of $S_{1}$ with $S_{1} / S_{3} \cong D_{3}$. So Hypothesis $3.1(\mathrm{~d})$ is satisfied.

Since every simple $\mathbb{F}_{2} \mathfrak{S}_{n}$-module is self-dual, also Hypothesis $3.1(\mathrm{~b})$ is satisfied. It remains to verify that Hypotheses $3.1(\mathrm{e})$ are satisfied. To this end, let $i \in$ $\{0, \ldots, t-1\}$. Then

$$
\begin{aligned}
{\left[S_{3 i+1}: S_{3(i+1)+1}\right] } & =\left[\bigwedge^{2}\left(M_{2^{i}}\right) / 2^{i}: \bigwedge^{2}\left(M_{2^{i+1}}\right) / 2^{i+1}\right]=\left[\bigwedge^{2}\left(M_{2^{i}}\right): \bigwedge^{2}\left(M_{2^{i+1}}\right) / 2\right] \\
& =\frac{\left[\bigwedge^{2}\left(M_{2^{i}}\right): \bigwedge^{2}\left(M_{2^{i+1}}\right)\right]}{2^{\left(\begin{array}{c}
n-1 \\
2
\end{array}\right)}}=2^{(n-2)\left(\begin{array}{c}
n-2 \\
1
\end{array}\right)-\left(\begin{array}{c}
n-1 \\
2
\end{array}\right)} \\
& =2^{\left(\begin{array}{c}
n-2 \\
2
\end{array}\right)}=2^{\left(\begin{array}{c}
n-1 \\
2
\end{array}\right)-(n-2)}=2^{d_{2}+d_{3}}
\end{aligned}
$$

by [5, Lemma 4.4, Proposition 2.5] and 3.5(b). Thus $S_{3 i+1} / S_{3(i+1)+1}$ has $\mathbb{F}_{2^{-}}$ dimension $d_{2}+d_{3}$, and $S_{3(i+1)+1} / 2 S_{3 i+1}$ has $\mathbb{F}_{2}$-dimension $\left(\begin{array}{c}n-1 \\ 2\end{array}\right)-d_{2}-d_{3}=d_{1}$.

Next suppose that $i \in\{1, \ldots, t-1\}$. We need to show that $S_{3 i+1} / 2 S_{3 i+1}$ is semisimple as $\mathbb{F}_{2} \mathfrak{S}_{n}$-module. By 3.5 , we know that $M_{2^{i}} / 2 M_{2^{i}} \cong D_{1} \oplus D_{2}$, as $\mathbb{F}_{2}$-modules. Moreover, using $[5,4.1(\mathrm{~d})]$ and $[4,(12.2)]$, we also know that

$$
\begin{aligned}
S_{3 i+1} / 2 S_{3 i+1} & \cong \bigwedge^{2}\left(M_{2^{i}}\right) / 2 \bigwedge^{2}\left(M_{2^{i}}\right) \cong \bigwedge^{2}\left(M_{2^{i}} / 2 M_{2^{i}}\right) \\
& \cong \bigwedge^{2}\left(D_{2}\right) \oplus\left(D_{1} \otimes D_{2}\right) \oplus \bigwedge^{2}\left(D_{1}\right) \cong D_{1} \oplus \bigwedge^{2}\left(D_{1}\right)
\end{aligned}
$$


as $\mathbb{F}_{2} \mathfrak{S}_{n}$-modules. Consequently, $\bigwedge^{2}\left(D_{1}\right)$ must have composition factors $D_{2}$ and $D_{3}$. On the other hand, recall that $D_{1}$ is isomorphic to the head of $S_{\mathbb{F}_{2}}^{(n-1,1)}$. The resulting $\mathbb{F}_{2} \mathfrak{S}_{n}$-epimorphism $S_{\mathbb{F}_{2}}^{(n-1,1)} \rightarrow D_{1}$ gives rise to an $\mathbb{F}_{2} \mathfrak{S}_{n}$-epimorphism $S_{\mathbb{F}_{2}}^{\left(n-2,1^{2}\right)} \cong \bigwedge^{2}\left(S_{\mathbb{F}_{2}}^{(n-1,1)}\right) \rightarrow \bigwedge^{2}\left(D_{1}\right)$. But we have already seen above that the only factor module of $S_{\mathbb{F}_{2}}^{\left(n-2,1^{2}\right)}$ with composition factors $D_{2}$ and $D_{3}$ has to be semisimple. Hence $\bigwedge^{2}\left(D_{1}\right) \cong D_{2} \oplus D_{3}$, and we have verified Hypothesis 3.1(e)(ii).

Lastly, we have $M_{2^{t}} \cong\left(S_{\mathbb{Z}_{2}}^{(n-1,1)}\right)^{*}$, and thus, by [5, 4.1(c)], also

$$
S_{3 t+1} \cong \bigwedge^{2}\left(M_{2^{t}}\right) \cong\left(\bigwedge^{2}\left(S_{\mathbb{Z}_{2}}^{(n-1,1)}\right)\right)^{*} \cong\left(S_{\mathbb{Z}_{2}}^{\left(n-2,1^{2}\right)}\right)^{*} \cong S_{1}^{*}
$$

as $\mathbb{Z}_{2} \mathfrak{S}_{n}$-lattices.

Therefore, $S_{1}, S_{4}, \ldots, S_{3 t+1}$ indeed satisfy Hypotheses 3.1 , and the proof of the lemma is complete.

In consequence of Lemma 3.6 we can now apply Theorem 3.2 to obtain

Theorem 3.7. Let $n>4$ be such that $n \equiv 0(\bmod 4)$, let $t:=\nu_{2}(n)$, and keep the notation from Lemma 3.6. Then the lattice of full-rank sublattices of the $\mathbb{Z}_{2} \mathfrak{S}_{n}$ lattice $S_{\mathbb{F}_{2}}^{\left(n-2,1^{2}\right)}$ is given by (7). The lattices $S_{1}, S_{2}, \ldots, S_{3 t+1}$ are representatives of the isomorphism classes of $\mathbb{Z}_{2}$-forms of the simple $\mathbb{Q}_{2} \mathfrak{S}_{n}$-module $S_{\mathbb{Q}_{2}}^{\left(n-2,1^{2}\right)}$.

Proof. By Lemma 3.6 and Theorem 3.2, we conclude that (7) is part of the lattice of full-rank sublattices of $S_{1}=S_{\mathbb{Z}_{2}}^{\left(n-2,1^{2}\right)}$ and that $S_{1}, S_{2}, \ldots, S_{3 t+1}$ are pairwise non-isomorphic $\mathbb{Z}_{2}$-forms of $S_{\mathbb{Q}_{2}}^{\left(n-2,1^{2}\right)}$. Let $\varepsilon$ be the block idempotent of $\mathbb{Q}_{2} \mathfrak{S}_{n}$ corresponding to $S_{\mathbb{Q}_{2}}^{\left(n-2,1^{2}\right)}$, and let $\Lambda$ the the graduated $\mathbb{Z}_{2}$-order $\varepsilon \mathbb{Z}_{2} \mathfrak{S}_{n} \varepsilon$ in $\varepsilon \mathbb{Q}_{2} \mathfrak{S}_{n} \varepsilon$. By Theorem 3.2, we also know that the exponent matrix of $\Lambda$ with respect to $S_{1}$ equals

$$
M:=\left(m_{i j}\right):=\left(\begin{array}{ccc}
0 & 0 & 0 \\
t & 0 & a \\
t & b & 0
\end{array}\right),
$$

for some $a, b \in \mathbb{N}$. In order to complete the proof of the theorem, it suffices to show that $a=b=1$ or, equivalently, that each of the lattices $S_{2}$ and $S_{3}$ has a unique maximal sublattice, namely the common maximal sublattice $S_{4}$. We examine $S_{2}$. By construction, $S_{2}$ is the unique maximal sublattice of $S_{1}$ such that $S_{1} / S_{2} \cong D_{2} \cong \mathbb{F}_{2}$. Therefore, $S_{2}$ has to be the $\mathbb{Z}_{2} \mathfrak{S}_{n}$-sublattice of $S_{1}$ constructed in [5, Lemma 7.5]; in particular, $S_{2}$ does not have a maximal sublattice $T$ such that $S_{2} / T \cong D_{2}$, by [5, Lemma 7.7(a)]. Let $L \subseteq S_{1}$ be the unique sublattice of $S_{1}$ such that $L \nsubseteq 2 S_{1}$ and $L / \operatorname{Rad}(L) \cong D_{3}$. Then, due to the structure of $M$, we must have 
$S_{1} \neq L \nsubseteq S_{3}$. Hence $L \subseteq S_{2}$, since $S_{2}$ and $S_{3}$ are the only maximal sublattices of $S_{1}$. If $L \neq S_{2}$, then $a>1$ and so there would be a maximal sublattice $T$ of $S_{2}$ such that $S_{2} / T \cong D_{2}$, a contradiction. Therefore, $L=S_{2}$ and $a=1$.

Since $S_{\mathbb{Q}_{2}}^{\left(n-2,1^{2}\right)}$ is a self-dual $\mathbb{Q}_{2} \mathfrak{S}_{n^{-}}$-module and $D_{1}, D_{2}, D_{3}$ are self-dual $\mathbb{F}_{2} \mathfrak{S}_{n^{-}}$ modules, (6) gives

$$
0+a+t=m_{12}+m_{23}+m_{31}=m_{21}+m_{32}+m_{13}=t+b+0,
$$

that is, $b=a=1$ as well. So, by Theorem $3.2(\mathrm{f}), S_{4}$ is also the unique maximal sublattice of $S_{3}$, and the assertion of the theorem follows.

Remark 3.8. For completeness, we also comment on the simple $\mathbb{Q}_{2} \mathfrak{S}_{4}$-module $S_{\mathbb{Q}_{2}}^{\left(2,1^{2}\right)}$. It is well known that, for every $r \in\{0, \ldots, n-1\}$ and every prime $p$, one has $S_{\mathbb{Q}_{p}}^{\left(r+1,1^{n-r-1}\right)} \cong S_{\mathbb{Z}_{p}}^{\left(n-r, 1^{r}\right)} \otimes \operatorname{sgn}_{\mathbb{Q}_{p}}$, where $\operatorname{sgn}_{\mathbb{Q}_{p}}$ denotes the one-dimensional sign module of $\mathbb{Q}_{p} \mathfrak{S}_{n}$; see [9, Theorem 6.7].

Thus, in particular, if $M_{1}, M_{2}, M_{3}$ denote the $\mathbb{Z}_{2} \mathfrak{S}_{4}$-sublattices of $S_{\mathbb{Z}_{2}}^{(3,1)}$ mentioned in 3.5, then $M_{1} \otimes \operatorname{sgn}_{\mathbb{Z}_{2}}, M_{2} \otimes \operatorname{sgn}_{\mathbb{Z}_{2}}$ and $M_{3} \otimes \operatorname{sgn}_{\mathbb{Z}_{2}}$ are representatives of the isomorphism classes of $\mathbb{Z}_{2}$-forms of $S_{\mathbb{Q}_{2}}^{\left(2,1^{2}\right)}$.

Together with [5, Theorem 1.1, Corollary 3.4] we now also have the following immediate corollary:

Corollary 3.9. Let $n \geqslant 4$, and let $V \in\left\{S_{\mathbb{Q}}^{\left(n-2,1^{2}\right)}, S_{\mathbb{Q}}^{\left(3,1^{n-3}\right)}\right\}$. Let $h(V)$ be the number of isomorphism classes of $\mathbb{Z}$-forms of $V$, and let $d(n)$ be the number of divisors of $n$ in $\mathbb{N}$. Then one has

$$
h(V)= \begin{cases}3 d(n) & \text { if } 2 \nmid n, \\ 2 d(n) & \text { if } n \equiv 2 \quad(\bmod 4), \\ 3 & \text { if } n=4, \\ \frac{\left(3 \nu_{2}(n)+1\right) d(n)}{\nu_{2}(n)+1} & \text { if } n \equiv 0 \quad(\bmod 4), n>4 .\end{cases}
$$

3.3. Application II: projective special linear groups. Our second application of Theorem 3.2 will involve the Steinberg module of the projective special linear group $\operatorname{PSL}_{2}(q)$ over suitable local fields of characteristic 0 . We begin by setting up the necessary notation.

3.10. Two-fold transitive permutation lattices. (a) Let $R$ be a principal ideal domain with field of fractions $K$ of characteristic 0 . Moreover, let $r \in \mathbb{N}$ with $r>2$ and let $G$ be a finite group acting two-transitively on a set $\Omega:=\left\{\omega_{1}, \ldots, \omega_{r}\right\}$. Let further $M_{R}$ be the corresponding permutation $R G$-lattice with $R$-basis $\Omega$, and 
let

$$
L_{R}:={ }_{R}\left\langle\omega_{2}-\omega_{1}, \ldots, \omega_{r}-\omega_{1}\right\rangle,
$$

which is an $R G$-sublattice of $M_{R}$ of rank $r-1$. Then the $K G$-module $V_{K}:=K L_{R}$ is absolutely simple, and $K M_{R} \cong V_{K} \oplus K$; see [7, Satz V.20.2]. Since $K M_{R}$ and $K$ are a self-dual $K G$-modules, so is $V_{K}$. Note that we also have $M_{R} \cong R \otimes_{\mathbb{Z}} M_{\mathbb{Z}}$ and $L_{R} \cong R \otimes_{\mathbb{Z}} L_{\mathbb{Z}}$.

(b) Now suppose that $K$ is a finite extension of the field $\mathbb{Q}_{p}$ of $p$-adic numbers. Let $R$ be the valuation ring of $K$ with respect to the extension of the $p$-adic valuation, and let $\mathfrak{m}=(\pi)$ be the maximal ideal in $R$.

Suppose that $p \mid r$, and let $t \in \mathbb{N}$ be such that $(r)=\mathfrak{m}^{t}=\left(\pi^{t}\right)$. For $j \in\{0, \ldots, t\}$, the map $\iota_{j}: L_{R} / \pi^{j} L_{R} \rightarrow M_{R} / \pi^{j} M_{R}, x+\pi^{j} L_{R} \mapsto x+\pi^{j} M_{R}$ is easily checked to be an injective homomorphism of $R G$-modules and $R /\left(\pi^{j}\right)[G]$-modules. Since $\pi^{j} \mid r$, the element $\sum_{i=1}^{r} \omega_{i}+\pi^{j} M_{R}$ spans a trivial submodule of $M_{R} / \pi^{j} M_{R}$, and so $\sum_{i=2}^{r}\left(\omega_{i}-\omega_{1}\right)+\pi^{j} L_{R}$ spans a trivial submodule of $L_{R} / \pi^{j} L_{R}$. The preimage of the latter under the canonical surjective $R G$-homomorphism $L_{R} \rightarrow L_{R} / \pi^{j} L_{R}$ is

$$
M_{\pi^{j}}:={ }_{R}\left\langle\sum_{i=2}^{r}\left(\omega_{i}-\omega_{1}\right)\right\rangle+\pi^{j} L_{R} \subseteq L_{R} ;
$$

in particular, $M_{\pi^{0}}, M_{\pi}, \ldots, M_{\pi^{t}}$ are $R G$-sublattices of $L_{R}$. As well, $M_{\pi^{j}}$ has $R$ basis $\left(\sum_{i=2}^{r}\left(\omega_{i}-\omega_{1}\right), \pi^{j}\left(\omega_{3}-\omega_{1}\right), \ldots, \pi^{j}\left(\omega_{r}-\omega_{1}\right)\right)$, for $j \in\{0, \ldots, t\}$. In particular, $M_{\pi^{0}}, M_{\pi}, \ldots, M_{\pi^{t}}$ are $R$-forms of the $K G$-module $V_{K}$ not contained in $\pi L_{R}$. Thus, by [13, Proposition 2.3], they are pairwise non-isomorphic as $R G$-lattices.

Now suppose, in addition, that the factor module $D$ of $L_{R} / \pi L_{R}$ modulo the trivial submodule $D_{1}$ mentioned above is a non-trivial, simple $k G$-module. By Lemma 3.11 below, the $k G$-module $L_{R} / \pi L_{R}$ then has to be indecomposable. Hence, by [15, Theorem (VI.1)] and [13, Proposition 2.3], one deduces that there are precisely $t+1$ isomorphism classes of $R$-forms of $V_{K}$, and hence $M_{\pi^{0}}, \ldots, M_{\pi^{t}}$ are representatives of these.

In fact, in this case, the $k G$-modules $M_{\pi^{j}} / \pi M_{\pi^{j}}$, for $j \in\{1, \ldots, t-1\}$ are all semisimple, hence isomorphic to $D \oplus D_{1}$. Moreover, for $j \in\{0, \ldots, t-1\}$, one has $M_{\pi^{j}} / M_{\pi^{j+1}} \cong D$ as $k G$-modules. This can, for instance, be deduced from Plesken's results in [13, Theorem 3.22] and [12, Satz (I.6)], which we shall also recall in 4.7.

Note that, in this case, the permutation $k G$-module $k \Omega \cong M_{R} / \pi M_{R}$ has composition factors $D$ (with multiplicity 1 ) and $k$ (with multiplicity 2 ). Since $k \Omega$ and $k$ are self-dual, so is $D$. 
The following properties of the $R G$-lattices introduced above are certainly well known. They will be important in the proofs of Lemma 3.17 and Proposition 3.18 below, so we include their proofs here.

Lemma 3.11. In the notation of 3.10(a), let $R$ be a principal ideal domain with field of fractions $K$, let $\mathfrak{m}:=(\pi)$ be a maximal ideal in $R$, let $k:=R /(\pi)$ be the corresponding residue field. Then the $k G$-module $L_{R} / \pi L_{R}$ does not have a trivial factor module.

Proof. For $x \in \pi L_{R}$, let $\bar{x}:=x+\pi L_{R} \in L_{R} / \pi L_{R}$. Assume that $L_{R} / \pi L_{R}$ has a trivial factor module, and let $\varphi: L_{R} / \pi L_{R} \rightarrow k$ be a non-trivial $k G$-homomorphism. Since the elements $\overline{\omega_{i}-\omega_{1}}$, for $i \in\{2, \ldots, r\}$, form a $k$-basis of $L_{R} / \pi L_{R}$, there is some $j \in\{2, \ldots, r\}$ with $\varphi\left(\overline{\omega_{j}-\omega_{1}}\right) \neq 0$. For $g \in G$, we have $\varphi\left(g\left(\overline{\omega_{j}-\omega_{1}}\right)\right)=$ $g \varphi\left(\overline{\omega_{j}-\omega_{1}}\right)=\varphi\left(\overline{\omega_{j}-\omega_{1}}\right)$. Since $r>2$, there is some $i \in\{2, \ldots, r\}$ such that $i \neq j$. Since $G$ acts two-transitively on $\Omega$, there exist $g, h \in G$ such that $g\left(\omega_{j}, \omega_{1}\right)=$ $\left(\omega_{j}, \omega_{i}\right)$ and $h\left(\omega_{j}, \omega_{1}\right)=\left(\omega_{i}, \omega_{1}\right)$. We get

$$
\begin{aligned}
0 & \neq g \varphi\left(\overline{\omega_{j}-\omega_{1}}\right)=\varphi\left(\overline{\omega_{j}-\omega_{i}}\right)=\varphi\left(\overline{\omega_{j}-\omega_{1}}\right)-\varphi\left(\overline{\omega_{i}-\omega_{1}}\right) \\
& =\varphi\left(\overline{\omega_{j}-\omega_{1}}\right)-h \varphi\left(\overline{\omega_{j}-\omega_{1}}\right)=0,
\end{aligned}
$$

a contradiction.

Lemma 3.12. In the notation of 3.10(b), one has an $R G$-isomorphism $M_{\pi^{t}} \cong M_{1}^{*}$.

Proof. The permutation $K G$-module $M_{K}=K M_{R}$ carries its natural non-degenerate symmetric $G$-invariant bilinear form $\beta$ such that $\beta\left(\omega_{i}, \omega_{j}\right)=\delta_{i j}$, for $i, j \in\{1, \ldots, r\}$. Via restriction, $\beta$ induces a symmetric $G$-invariant bilinear form on $V_{K}$, and one easily checks that this is still non-degenerate. Let $N \subseteq V_{K}$ be any $R$-form of $V_{K}$, let $\left(b_{1}, \ldots, b_{r-1}\right)$ be an $R$-basis of $N$, and consider $N^{\#}:=\left\{v \in V_{K}: \beta(v, N) \subseteq R\right\}$. Then $N^{\#}$ is also an $R$-form of $V_{K}$. Moreover, $N \subseteq N^{\#}$ and, since $R$ is local with finite residue field $k$, one has

$$
\left[N^{\#}: N\right]=\mid R / \operatorname{det}\left(\left(\beta\left(b_{i}, b_{j}\right)\right)_{1, \leqslant i, j \leqslant r-1} \mid ;\right.
$$

see [5, Proposition 2.5]. Also note that the map $N^{\#} \rightarrow N^{*}, x \mapsto(y \mapsto \beta(x, y))$ defines an $R G$-isomorphism.

Next observe that we have $\pi^{t} M_{\pi^{t}} \subseteq M_{\pi^{t}} \subseteq M_{1} \subseteq \pi^{t} M_{\pi^{t}}^{\#}$, by the choice of $t$. So, in order to complete the proof of the lemma, it suffices to show that $\left[M_{1}\right.$ : $\left.\pi^{t} M_{\pi^{t}}\right]=\left[M_{\pi^{t}}^{\#}: M_{\pi^{t}}\right]$. For then we get $\left[M_{1}: \pi^{t} M_{\pi^{t}}\right]=\left[\pi^{t} M_{\pi^{t}}^{\#}: \pi^{t} M_{\pi^{t}}\right]$, and hence $M_{1}=\pi^{t} M_{\pi^{t}}^{\#} \cong M_{\pi^{t}}^{\#} \cong M_{\pi^{t}}^{*}$ as $R G$-lattices. 
So consider the $R$-basis $\left(b_{1}, \ldots, b_{r-1}\right):=\left(\omega_{2}-\omega_{1}, \ldots, \omega_{r}-\omega_{1}\right)$ of $M_{1}$ and the $R$-basis $\left(c_{1} \ldots, c_{r-1}\right):=\left(\sum_{i=2}^{r}\left(\omega_{i}-\omega_{1}\right), \pi^{t}\left(\omega_{3}-\omega_{1}\right), \ldots, \pi^{t}\left(\omega_{r}-\omega_{1}\right)\right)$ of $\pi^{t} M_{\pi^{t}}$. Then one has

$$
\operatorname{det}\left(\left(\beta\left(c_{i}, c_{i}\right)_{1 \leqslant i, j \leqslant r-1}\right)=\left(\pi^{t}\right)^{2(r-2)} \cdot \operatorname{det}\left(\left(\beta\left(b_{i}, b_{j}\right)_{1 \leqslant i, j \leqslant r-1}\right)=\left(\pi^{t}\right)^{2(r-2)} \cdot r,\right.\right.
$$

hence $\left[M_{\pi^{t}}^{\#}: M_{\pi^{t}}\right]=\left|R /\left(\left(\pi^{t}\right)^{2(r-2)} \cdot r\right)\right|=\left|R / r^{2 r-3} R\right|$. On the other hand, using $[5$, Proposition 2.5], we also see that $\left[M_{1}: \pi^{t} M_{\pi^{t}}\right]=\left|R /\left(\left(\pi^{t}\right)^{2(r-2)} \cdot \pi^{t}\right)\right|=\left|R / r^{2 r-3} R\right|$.

This completes the proof of the lemma.

3.13. (Projective) general linear and (projective) special linear groups. Keep the notation as in 3.10 .

(a) Let $n \in \mathbb{N}$ with $n>1$, let $p$ be a prime, and let $q$ be a power of a prime. The general linear group $\mathrm{GL}_{n}(q)$ acts two-transitively on the set of one-dimensional subspaces of $\mathbb{F}_{q}^{n}$; as above, we simply denote this set by $\Omega=\left\{\omega_{1}, \ldots, \omega_{r}\right\}$, where $r=$ $1+q+\cdots+q^{n-1}$. Suppose that $p \nmid q$. In the notation of 3.10 above, the $k\left[\operatorname{GL}_{n}(q)\right]-$ module $L_{R} / \pi L_{R}$ is absolutely simple if $p \nmid r$; see [10, p.16, p.47, Theorem 20.3] So, in this case $L_{R}$ is up to isomorphism the unique $R$-form of $V_{K}$; this follows from [4, Proposition (16.16)], see also [5, Proposition 2.12]. If $p \mid r$, then $L_{R} / p L_{R}$ has precisely two absolutely simple composition factors $D$ and $D_{1}$ satisfying the properties of $3.10(\mathrm{~b})$; see, for instance, [10, (11.12)(iii), Theorem 16.3, Theorem 20.7]. The same is true when replacing $\operatorname{GL}_{n}(q)$ by the projective general linear group $\mathrm{PGL}_{n}(q)$. So, in these cases, Plesken's result [15, Theorem (VI.1)] determines the $R$-forms of $V_{K}$ listed in (8).

(b) One has $\mathrm{SL}_{n}(q) \preccurlyeq \mathrm{GL}_{n}(q)$, and one can also regard $\mathrm{PSL}_{n}(q)$ as a normal subgroup of $\mathrm{PGL}_{n}(q)$ in the obvious way. Via restriction, the $K\left[\mathrm{GL}_{n}(q)\right]$-module $V_{K}$ in (a) becomes a $K\left[\mathrm{SL}_{n}(q)\right]$-module (and also a $K\left[\operatorname{PSL}_{n}(q)\right]$-module), which is still absolutely simple, since also $\mathrm{SL}_{n}(q)$ acts two-transitively on $\Omega$.

(c) In the following, we shall focus on the case $n=2$. Then $V_{K}$ is the Steinberg module of the (projective) general and (projective) special linear groups under consideration; see [1, Chapter 9]. Moreover, we shall from now on suppose that $K$ is a finite unramified extension of $\mathbb{Q}_{p}$ and $R$ is the valuation ring in $K$ with respect to the extension of the $p$-adic valuation. In this case we may take $\pi:=p$. The residue field $k$ is isomorphic to $\mathbb{F}_{p^{f}}$, where $f$ is the degree of $K$ over $\mathbb{Q}_{p}$. We shall use the results of Plesken in [15, Theorem (VI.1); Chapter VII] and Theorem 3.2 to obtain representatives of the isomorphism classes of $R$-forms of $\operatorname{Res}_{\mathrm{PSL}_{2}(q)}^{\mathrm{PGL}_{2}(q)}\left(V_{K}\right)$. 
Remark 3.14. (a) Keep the notation from 3.13(c). Suppose first that $p$ is odd and that $q$ is power of a prime different from $p$. Let $G:=\mathrm{PGL}_{2}(q)$ and $H:=\mathrm{PSL}_{2}(q)$. If $p$ does not divide the order of $H$, then the $k H$-module $\operatorname{Res}_{H}^{G}\left(L_{R} / p L_{R}\right)$ is absolutely simple. Thus, in this case, $\operatorname{Res}_{H}^{G}\left(L_{R}\right)$ is up to isomorphism the unique $R$-form of $\operatorname{Res}_{H}^{G}\left(V_{K}\right)$; see [4, Proposition (16.16)], [5, Proposition 2.12].

Hence, we may suppose that $p|| H \mid$. Recall that $|H|$ is a divisor of $(q+1) q(q-1)$. If $p \mid(q-1)$, then, by [1, Section 9.4.2], the $k H$-module $\operatorname{Res}_{H}^{G}\left(L_{R} / p L_{R}\right)$ is absolutely simple. So, also in this case, $\operatorname{Res}_{H}^{G}\left(L_{R}\right)$ is up to isomorphism the unique $R$-form of $\operatorname{Res}_{H}^{G}\left(V_{K}\right)$. If $p \mid(q+1)$, then it follows from [1, Section 9.4.3] that the $k H$-module $\operatorname{Res}_{H}^{G}\left(L_{R} / p L_{R}\right)$ has a trivial submodule with (absolutely) simple quotient. So, we are in the situation of 3.10(b), and representatives of the isomorphism classes of $R$-forms of $V_{K}$ are given by the RH-lattices in (8), for $t=\nu_{p}(q+1)$.

(b) Now let $p=2$, and let $q$ be odd. If $q \equiv \pm 3(\bmod 8)$ and if the degree of $K$ over $\mathbb{Q}_{2}$ is odd, then the $k H$-module $\operatorname{Res}_{H}^{G}\left(L_{R} / 2 L_{R}\right)$ has two composition factors; see [15, p. 110]. In consequence of 3.13(a) and Lemma 3.11, we are then again in the situation of 3.10(b), and representatives of the isomorphism classes of $R$-forms of $\operatorname{Res}_{H}^{G}\left(V_{K}\right)$ are given by the lattices in (8), for $t=\nu_{2}(q+1)$.

If $q \equiv \pm 1(\bmod 8)$, or if $q \equiv \pm 3(\bmod 8)$ and the degree of $K$ over $\mathbb{Q}_{2}$ is even, then $\operatorname{Res}_{H}^{G}\left(L_{R} / 2 L_{R}\right)$ has three (absolutely) simple composition factors; see [15, p. 110], [1, Section 9.4.4]. These cases will be dealt with in the following.

Remark 3.15. As for the case of equal characteristic, that is, in the case where $p \mid q$, note that the $k H$-module $\operatorname{Res}_{H}^{G}\left(L_{R} / p L_{R}\right)$ is projective and absolutely simple; see [1, Lemma 10.2.4]. So, also in this case, $\operatorname{Res}_{H}^{G}\left(L_{R}\right)$ is up to isomorphism the unique $R$-form of $\operatorname{Res}_{H}^{G}\left(V_{K}\right)$.

Hypotheses 3.16. For the remainder of this subsection, we suppose that $q$ is an odd prime power and $p=2$. We set $G:=\operatorname{PGL}_{2}(q)$, and let $H:=\operatorname{PSL}_{2}(q)$. Let $(K, R, k)$ be as in $3.13(\mathrm{c})$. If $q \equiv \pm 1(\bmod 8)$, then we may take $K$ to be any finite unramified extension of $\mathbb{Q}_{2}$. If $q \equiv \pm 3(\bmod 8)$, then let $K$ be a finite unramified extension of $\mathbb{Q}_{2}$ of even degree; in particular, $k$ then contains the field with four elements. Lastly, let $V_{K}$ be the absolutely simple $K G$-module with $R$-form $L_{R}$ as defined in $3.13(\mathrm{a})$.

Lemma 3.17. Let $q$ be an odd prime power, and let $t:=\nu_{2}(q+1)$. With Hypotheses 3.16 one has the following:

(a) The $k H$-module $\operatorname{Res}_{H}^{G}(L / 2 L)$ has precisely three composition factors $D_{1}, D_{2}$ and $D_{3}$, all of which are absolutely simple and pairwise non-isomorphic. 
More precisely, $D_{1} \cong k$, and $\operatorname{Res}_{H}^{G}(D) \cong D_{2} \oplus D_{3}$; in particular $\operatorname{dim}_{k}\left(D_{2}\right)=$ $(q-1) / 2=\operatorname{dim}_{k}\left(D_{3}\right)$.

(b) For $i \in\{0, \ldots, t\}$, let $M_{2^{i}} \subseteq L_{R}$ be the $R G$-lattice in (8), and let $S_{3 i+1}:=$ $\operatorname{Res}_{H}^{G}\left(M_{2^{i}}\right)$. Then $S_{1}, S_{4}, \ldots, S_{3 t+1}$ are $R$-forms of $\operatorname{Res}_{H}^{G}\left(V_{K}\right)$ satisfying Hypotheses 3.1.

Proof. Assertion (a) is well known; see, for instance, [1, Section 9.4.4] and [15, p.110]. Note that $D_{2}$ is $G$-conjugate to $D_{3}$. As for assertion (b), note first that $S_{1}, S_{4}, \ldots, S_{3 t+1}$ are of course $R$-forms of $\operatorname{Res}_{H}^{G}\left(V_{K}\right)$ satisfying Hypothesis 3.1(e)(i). Recall from 3.10(b) and 3.13(a) that $M_{2^{i}} / 2 M_{2^{i}} \cong D_{1} \oplus D$, for $i \in\{1, \ldots, t-1\}$, and that $M_{2^{i}} / M_{2^{i+1}} \cong D$, for $i \in\{0, \ldots, t-1\}$, as $k G$-modules. Hence $S_{1}, S_{4}, \ldots, S_{3 t+1}$ satisfy Hypothesis 3.1(e)(ii). By Lemma 3.12, also Hypothesis 3.1(e)(iii) is satisfied.

Since the simple $k G$-module $D$ is self-dual and since $\operatorname{Res}_{H}^{G}(D) \cong D_{2} \oplus D_{3}$, Hypotheses 3.1(b) are satisfied. Setting $d_{i}:=\operatorname{dim}_{k}\left(D_{i}\right)$, for $i \in\{1,2,3\}$, we have $d_{2}=d_{3}=(q-1) / 2 \neq 1=d_{1}$ and $d_{1} \neq d_{2}+d_{3}$.

It remains to verify the assertion on the submodule structure of the $\mathrm{kH}$-module $\operatorname{Res}_{H}^{G}(\bar{L}):=\operatorname{Res}_{H}^{G}\left(L_{R} / 2 L_{R}\right)$. We know that $\bar{L}$ has a trivial submodule $U$ with factor module isomorphic to $\operatorname{Res}_{H}^{G}(D) \cong D_{2} \oplus D_{3}$; in particular, $\operatorname{Rad}\left(\operatorname{Res}_{H}^{G}(\bar{L})\right) \subseteq$ $U$. By Lemma 3.11, we also know that $\operatorname{Res}_{H}^{G}(\bar{L})$ does not have a trivial factor module; in particular, $\operatorname{Res}_{H}^{G}(\bar{L})$ cannot be semisimple, implying $\operatorname{Rad}\left(\operatorname{Res}_{H}^{G}(\bar{L})\right)=$ $U$. Therefore, $\operatorname{Res}_{H}^{G}(\bar{L})$ has precisely two maximal submodules, $U_{2}$ and $U_{3}$, where $U_{2}$ has composition factors $D_{2}$ and $D_{1}$, and $U_{3}$ has composition factors $D_{1}$ and $D_{3}$. So $U_{2}$ has to be $G$-conjugate to $U_{3}$. Since $\operatorname{Res}_{H}^{G}(\bar{L})$ is not semisimple, both $U_{2}$ and $U_{3}$ are indecomposable, and the common trivial submodule of $U_{2}$ and $U_{3}$ is the unique simple submodule of $\operatorname{Res}_{H}^{G}(\bar{L})$. Thus $\operatorname{Soc}\left(\operatorname{Res}_{H}^{G}(\bar{L})\right)=\operatorname{Rad}\left(\operatorname{Res}_{H}^{G}(\bar{L})\right) \cong k \cong D_{1}$. This completes the proof of the lemma.

Proposition 3.18. Let $q$ be an odd prime power, and let $t:=\nu_{2}(q+1)$. In the notation of Lemma 3.17, the RH-lattice $L_{R}$ has submodule lattice (7). The lattices $S_{1}, S_{2}, \ldots, S_{3 t+1}$ are representatives of the isomorphism classes of $R$-forms of the absolutely simple $K H$-module $\operatorname{Res}_{H}^{G}\left(V_{K}\right)$.

Proof. Let $\varepsilon$ be the block idempotent of $K H$ corresponding to the absolutely simple module $\operatorname{Res}_{H}^{G}\left(V_{K}\right)$. Consider the graduated $R$-order $\Lambda:=\varepsilon R H \varepsilon$ in $\varepsilon K H \varepsilon$. By Lemma 3.17 and Theorem 3.2, we know that (7) is part of the lattice of full-rank sublattices of $L_{R}=S_{1}$. Moreover, $S_{1}, S_{2}, \ldots, S_{3 t+1}$ are pairwise non-isomorphic $R$-forms of the $K H$-module $V_{K}$. On the other hand, by [15, Chapter VII], there is a $K H$-lattice $L^{\prime} \subseteq V_{K}$ that is an $R$-form of $V_{K}$ and the exponent matrix of $\Lambda$ with 
respect to $L^{\prime}$ is

$$
M_{L^{\prime}}=\left(\begin{array}{lll}
0 & t & t \\
0 & 0 & 1 \\
0 & 1 & 0
\end{array}\right) .
$$

From this and 2.6(c) one easily deduces that the isomorphism classes of $R$-forms of the $K H$-module $V_{K}$ are in bijection with the set

$$
\{(0,0,0),(j, 0,0),(j, 0,1),(j, 1,0): j \in\{1, \ldots, t\}\},
$$

which has cardinality $3 t+1$. Hence, $(7)$ has to be the complete lattice of full-rank sublattices of $L_{R}=S_{1}$, and $S_{1}, S_{2}, \ldots, S_{3 t+1}$ are representatives of the isomorphism classes of $R$-forms of the $K H$-module $V_{K}$.

Question 3.19. To conclude this section, suppose again that $G$ is any finite group acting two-transitively on a finite set $\Omega=\left\{\omega_{1}, \ldots, \omega_{r}\right\}$ with $r>2$. In the notation of 3.10, we know that the absolutely simple $K G$-module $V_{K}$ admits at least $t+1$ pairwise non-isomorphic $R$-forms, representatives of which are given by the $R G$ lattices $L_{R}=M_{\pi^{0}}, \ldots, M_{\pi^{t}}$ in (8). We also know, by Lemma 3.12, that $M_{\pi^{t}}$ is isomorphic to the dual lattice $M_{1}^{*}=L_{R}^{*}$. Consider the $k G$-module $L_{k}:=L_{R} / \pi L_{R}$ and its trivial submodule $D_{1}$ as in 3.10. Suppose that $L_{k} / D_{1}$ is the direct sum of two non-trivial pairwise non-isomorphic simple $k G$-modules $D_{2}$ and $D_{3}$. For $i \in\{0, \ldots, t\}$, set $S_{3 i+1}:=M_{\pi^{i}}$.

Do these $R G$-lattices then satisfy Hypotheses 3.1 ? If so, is $S_{4}$ the only maximal $R G$-sublattice of each of the maximal sublattices $S_{2}$ and $S_{3}$ of $S_{1}$ ? If this was the case, then Theorem 3.2 would be applicable to determine representatives of the isomorphism classes of $R$-forms of $V_{K}$, generalizing Proposition 3.18.

\section{Zeta functions}

In this section we briefly review the notion of zeta functions of modules. We follow Solomon [16], who introduced these objects to study enumerative problems in integral representation theory. After introducing the general zeta function, we focus on the case over local principal ideal domains and determine zeta functions of various types of lattices, including the ones from Section 3.

\subsection{Local and global zeta functions.}

Notation 4.1. Let $R$ be a unitary ring, and let $M$ be a left $R$-module such that, for all $n \in \mathbb{N}$, the number $a_{n}$ of $R$-sublattices of $M$ with index $n$ is finite. One 
defines the zeta function of $M$ by

$$
\zeta_{R}(M, s):=\sum_{n=1}^{\infty} a_{n} n^{-s}, \text { where } s \in \mathbb{C} .
$$

We view this as a formal Dirichlet series and ignore questions of convergence. Note that if $M$ is a free $\mathbb{Z}$-module of finite rank $r$, then, by comparing $\zeta_{R}(M, s)$ with $\zeta_{\mathbb{Z}}(M, s)$, one sees that $\zeta_{R}(M, s)$ converges absolutely for $\operatorname{Re}(s)>r$; see also [2].

4.2. Local zeta functions. (a) Let $R$ be a local principal ideal domain with maximal ideal $\mathfrak{m}=(\pi)$, field of fractions $K$ and finite residue field $k=R / \mathfrak{m}$ of cardinality $q$. Assume that $\Lambda$ is an $R$-order and $M$ a $\Lambda$-lattice. Since $k$ is finite, the number of $\Lambda$-sublattices of $M$ with bounded index is finite. Moreover, as the indices of such $\Lambda$-sublattices of $M$ must be powers of $q$, there exists some $Z(M) \in \mathbb{Z}[[X]]$ such that $Z(M)\left(q^{-s}\right)=\zeta_{\Lambda}(M, s)$. By defining $(M: N):=X^{i}$, for $i \in \mathbb{N}_{0}$ and every $R$-submodule of $N$ of $M$ with $[M: N]=q^{i}$, this can be rewritten as

$$
Z(M)=\sum_{N \subseteq M}(M: N),
$$

where the sum is taken over all $\Lambda$-sublattices $N$ of $M$ such that $[M: N]<\infty$.

(b) Assume that, up to isomorphism, there are only finitely many $\Lambda$-sublattices $M=M_{1}, M_{2}, \ldots, M_{r}$ of $M$ with finite index, for some $r \in \mathbb{N}$. For $j \in\{1, \ldots, r\}$, one defines

$$
Z\left(M, M_{j}\right)=\sum_{\substack{N \subseteq M \\[M: N]<\infty \\ N \cong M_{j}}}(M: N) .
$$

Analogously, one defines $Z\left(M_{i}, M_{j}\right)$, for all $i, j \in\{1, \ldots, r\}$. Having fixed an ordering on $M_{1}, \ldots, M_{r}$, the matrix $\left(Z\left(M_{i}, M_{j}\right)\right)_{1 \leqslant i, j \leqslant r} \in \mathbb{Z}[[X]]^{r \times r}$ is uniquely determined by $M$, and we shall from now on denote it by $\mathbf{B}_{M}$. Note that $Z\left(M_{i}\right)$ is the sum of the entries of the $i$ th row of $\mathbf{B}_{M}$, for $i \in\{1, \ldots, r\}$.

(c) We denote by $\max (M)$ the set of maximal $\Lambda$-sublattices of $M$, and by $\operatorname{Rad}(M)=\bigcap_{N \in \max (M)} N$ again the Jacobson radical of $M$. Furthermore, we consider the following sets of $\Lambda$-sublattices of $M: \Phi(M):=\{N: \operatorname{Rad}(M) \subseteq N \subseteq M\}$ and $\Phi(M, L):=\{N \in \Phi(M): N \cong L\}$. Finally, for $L \in \Phi(M)$, let $\mu(M, L):=$ $\sum_{J}(-1)^{|J|}$, where the sum runs over all subsets $J \subseteq \max (M)$ with $\bigcap_{N \in J} N=L$. The matrix $\left(A_{i j}\right)_{1 \leqslant i, j \leqslant r} \in \mathbb{Z}[X]^{r \times r}$ defined by

$$
A_{i j}=\sum_{L \in \Phi\left(M_{i}, M_{j}\right)} \mu\left(M_{i}, L\right)\left(M_{i}: L\right)
$$


is uniquely determined by $M$, and we denote it by $\mathbf{A}_{M}$. By [16, Lemma 3], the matrix $\mathbf{A}_{M}$ is the inverse of $\mathbf{B}_{M}$.

4.3. Global zeta functions. Assume that $M$ is a $\mathbb{Z}$-form of a $\mathbb{Q} G$-module $V$, for which we want to determine the zeta function $\zeta_{\mathbb{Z} G}(M, s)$. Then, for every prime $p$, the $p$-adic completion $M_{p}:=\mathbb{Z}_{p} \otimes_{\mathbb{Z}} M$ is a $\mathbb{Z}_{p}$-form of the $\mathbb{Q}_{p} G$-module $V_{p}:=\mathbb{Q}_{p} \otimes_{\mathbb{Q}} V$, giving rise to a local zeta function $\zeta_{\mathbb{Z}_{p} G}\left(M_{p}, s\right)$. By 4.2, we know that

$$
\zeta_{\mathbb{Z}_{p} G}\left(M_{p}, s\right)=Z\left(M_{p}\right)\left(p^{-s}\right) .
$$

Let $\mathbb{P} \subset \mathbb{N}$ be the set of all prime numbers. By [16], one has

$$
\zeta_{\mathbb{Z} G}(M, s)=\prod_{p \in \mathbb{P}} \zeta_{\mathbb{Z}_{p} G}\left(M_{p}, s\right) .
$$

In [16] it is also shown that there exists a complex function

$$
\zeta_{V}(s)=\prod_{p \in \mathbb{P}} \zeta_{V, p}(s)
$$

depending only on $V$, such that $\zeta_{V, p}(s)=\zeta_{\mathbb{Z}_{p} G}\left(M_{p}, s\right)$, for all primes $p$ not dividing the group order $|G|$. In particular, if $P$ is a finite set of prime numbers containing all prime divisors of $|G|$, then

$$
\zeta_{\mathbb{Z} G}(M, s)=\zeta_{V}(s) \prod_{p \in P} \frac{\zeta_{\mathbb{Z}_{p} G}\left(M_{p}, s\right)}{\zeta_{V, p}(s)} .
$$

Thus, when determining the global zeta function $\zeta_{\mathbb{Z} G}(M, s)$, it is sufficient to determine $\zeta_{V}(s)$ as well as the local zeta functions $\zeta_{\mathbb{Z}_{p} G}\left(M_{p}, s\right)$, for all prime divisors of $|G|$. The task of determining $\zeta_{V}(s)$ is straightforward, once the structure of the blocks of $\mathbb{Q} G$ containing the indecomposable direct summands of $V$ are known; see [16, (1.2)]. If $V$ is absolutely simple of dimension $d$, then $\zeta_{V}(s)=\zeta_{\mathbb{Q}}(d s)$, where $\zeta_{\mathbb{Q}}$ is the Riemann zeta function. In particular, in this case, we have $\zeta_{V, p}(s)=$ $\left(1-p^{-d s}\right)^{-1}$, for all $p \in \mathbb{P}$.

Lastly, note that if $V$ is simple and if $p \in \mathbb{P}$ is such that the $\mathbb{F}_{p} G$-module $M / p M \cong M_{p} / p M_{p}$ is also simple, then $\zeta_{\mathbb{Z}_{p} G}\left(M_{p}, s\right)=\zeta_{V, p}(s)$. Namely, in this case, $p M_{p}$ is the unique maximal sublattice of $M_{p}$ and $\left\{p^{i} M_{p}: i \in \mathbb{N}_{0}\right\}$ is the set of all $\mathbb{Z}_{p} G$-sublattices of $M_{p}$. Hence

$$
\zeta_{\mathbb{Z}_{p} G}\left(M_{p}, s\right)=\sum_{i=0}^{\infty}\left(p^{i d}\right)^{-s}=\frac{1}{1-p^{-d s}}=\zeta_{V, p}(s)
$$


4.2. Uniserial reductions. Throughout this subsection, let $R$ be a local principal ideal domain with maximal ideal $\mathfrak{m}=(\pi)$, field of fractions $K$ and finite residue field $k=R / \mathfrak{m}$ of cardinality $q$. Assume further that $G$ is a finite group and $M$ is an $R$-form of an absolutely simple $K G$-module $V$ such that the lattice of $R G$ sublattices of $M$ of full $R$-rank is totally ordered. This happens, for instance, if the reduction modulo $\mathfrak{m}$ of every $R$-form of $V$ is a uniserial $k G$-module; see [5, Proposition 3.7].

Denote by $\pi M=M_{r+1} \subseteq M_{r} \subseteq \cdots \subseteq M_{1}=M$ a chain of $R$-forms of $V$, such that, for $i \in\{1, \ldots, r\}$, the module $M_{i+1}$ is a maximal $R G$-sublattice of $M_{i}$. By [13, Proposition 2.3], we know that $M_{1}, \ldots, M_{r}$ form a set of representatives of the $R$-forms of $V$.

Our next aim is to determine, for each $i \in\{1, \ldots, r\}$, the zeta function $\zeta_{R G}\left(M_{i}, s\right)$, by determining $\mathbf{B}_{M}$ and $Z\left(M_{i}\right) \in \mathbb{Z}[[X]]$. For $i \in\{1, \ldots, r\}$, we denote by $d_{i}$ the $k$-dimension of $M_{i} / M_{i+1}$, and we set $d:=d_{1}+\cdots+d_{r}$.

Lemma 4.4. With the above notation, the matrix $\mathbf{A}_{M}=\left(A_{i j}\right) \in \mathbb{Z}[X]^{r \times r}$ is given by

$$
A_{i j}= \begin{cases}1 & \text { if } i=j, \\ -X^{d_{i}} & \text { if } i \neq r, j=i+1, \\ -X^{d_{r}} & \text { if } i=r, j=1 \\ 0 & \text { otherwise. }\end{cases}
$$

Thus

$$
\mathbf{A}_{M}=\left(\begin{array}{cccccc}
1 & -X^{d_{1}} & 0 & 0 & \ldots & 0 \\
0 & 1 & -X^{d_{2}} & 0 & \ldots & 0 \\
\vdots & \vdots & \vdots & \vdots & \vdots & \vdots \\
0 & 0 & 0 & 0 & 1 & -X^{d_{r-1}} \\
-X^{d_{r}} & 0 & 0 & 0 & 0 & 1
\end{array}\right)
$$

Proof. Since the lattice of full-rank $R G$-sublattices of $M$ is totally ordered, we have $\max \left(M_{i}\right)=\left\{M_{i+1}\right\}$, for $i \in\{1, \ldots, r-1\}$, and $\max \left(M_{r}\right)=\left\{\pi M_{1}\right\}$. Thus $\Phi\left(M_{i}\right)=$ $\left\{M_{i}, M_{i+1}\right\}$, for $i \in\{1, \ldots, r-1\}$, and $\Phi\left(M_{r}\right)=\left\{M_{r}, \pi M_{1}\right\}$. In particular,

$$
\Phi\left(M_{i}, M_{j}\right)= \begin{cases}\left\{M_{j}\right\} & \text { if } i \neq r, j \in\{i, i+1\}, \\ \left\{M_{j}\right\} & \text { if } i=r, j \in\{1, r\}, \\ \emptyset & \text { otherwise. }\end{cases}
$$

For $i, j \in\{1, \ldots, r\}$ and $L \in \Phi\left(M_{i}, M_{j}\right)$, we have $\mu\left(M_{i}, L\right)=1$ if $L=M_{i}$, and $\mu\left(M_{i}, L\right)=-1$ otherwise. Thus the claim follows. 
Proposition 4.5. The matrix $\mathbf{B}=\left(B_{i j}\right)_{1 \leqslant i, j \leqslant r}$ defined by

$$
B_{i j}=\frac{1}{1-X^{d}} \begin{cases}X^{d_{i}+\cdots+d_{j-1}} & \text { if } j \geqslant i, \\ X^{d} B_{j i}^{-1} & \text { if } j<i\end{cases}
$$

satisfies $\mathbf{B}=\mathbf{B}_{M}$.

Proof. It is sufficient to show that $\mathbf{B} \mathbf{A}_{M}$ is the identity matrix. To this end, let $A_{j}$ be the $j$ th column of $\mathbf{A}_{M}$ and $B_{i}$ the $i$ th row of $\mathbf{B}$. Then

$$
\begin{aligned}
& \left(1-X^{d}\right) \cdot B_{j} \cdot A_{1} \\
= & B_{j 1}-X^{d_{r}} B_{j r} \\
= & \begin{cases}1-X^{d_{r}} B_{1 r}=1-X^{d} & \text { if } j=1, \\
X^{d} B_{1 j}^{-1}-X^{d_{r}} B_{j r}=X^{d_{j}+\cdots+d_{r}}-X^{d_{j}+\cdots+d_{r}} & \text { if } 1<j<r \\
B_{r 1}-X^{d_{r}} B_{r r}=X^{d_{r}}-X^{d_{r}} & \text { if } j=r\end{cases} \\
= & \begin{cases}1-X^{d} & \text { if } j=1 \\
0 & \text { otherwise. }\end{cases}
\end{aligned}
$$

Now let $i>1$ and consider $\left(1-X^{d}\right) B_{j} \cdot A_{i}=B_{j, i-1} \cdot\left(-X^{d_{i-1}}\right)+B_{j i}$. If $j<i-1$, then

$$
\left(1-X^{d}\right) B_{j} \cdot A_{i}=X^{\sum_{k=j}^{i-2} d_{k}}\left(-X^{d_{i-1}}\right)+X^{\sum_{k=j}^{i-1} d_{k}}=0
$$

if $j=i-1$, then

$$
\left(1-X^{d}\right) B_{j} \cdot A_{i}=-X^{d_{i-1}}+B_{i-1, i}=-X^{d_{i-1}}+X^{d_{i-1}}=0 ;
$$

and if $j>i$, then

$$
\left(1-X^{d}\right) B_{j} \cdot A_{i}=-X^{d_{i-1}} X^{d} B_{i-1, j}^{-1}+X^{d} B_{i, j}^{-1}=0,
$$

since $B_{i, j} / B_{i-1, j}=X^{d_{i-1}}$. Finally, if $j=i$, then

$$
\left(1-X^{d}\right) B_{i} \cdot A_{i}=B_{i, i-1}\left(-X^{d_{i-1}}\right)+1=X^{d} B_{i-1, i}^{-1}\left(-X^{d_{i-1}}\right)=1-X^{d} .
$$

By summing up the entries of $\mathbf{B}_{M}$ row-wise, we obtain:

Corollary 4.6. For $i \in\{1, \ldots, r\}$, one has

$$
Z\left(M_{i}\right)=\frac{1}{1-X^{d}}\left(\sum_{j=1}^{i-1} X^{d-d_{j}-\cdots-d_{i-1}}+\sum_{j=i}^{r} X^{d_{i}+\cdots+d_{j-1}}\right)
$$




\subsection{Modular reductions with two non-isomorphic composition factors.}

In this section, let $R$ be a local principal ideal domain with maximal ideal $\mathfrak{m}=(\pi)$, field of fractions $K$, and finite residue field $k=R / \mathfrak{m}$ of cardinality $q$. Let $V$ be an absolutely simple $K G$-module of dimension $d$ such that the reduction modulo $\mathfrak{m}$ of any $R$-form of $V$ has two non-isomorphic composition factors $D_{1}$ and $D_{2}$. Assume further that the Jordan-Zassenhaus theorem holds for $R$-forms of $V$, that is, up to isomorphism there are only finitely many $R$-forms of $V$. By [12, Satz (I.6)], there exists an $R$-form $M$ of $V$ such that $M / \pi M$ is indecomposable. We fix such an $R$-form $M$ of $V$, for the remainder of this subsection. We shall suppose that the head of $M / \pi M$ is isomorphic to $D_{1}$. In fact, [12, Satz (I.6)] is stated in the case where $R=\mathbb{Z}$ and $\mathfrak{m}$ is any maximal ideal in $\mathbb{Z}$. The proof, however, generalizes literally to our situation. Alternatively, see also [13, Theorem 3.22].

4.7. The submodule lattice of $M$. Now let $t+1$ be the number of isomorphism classes of $R$-forms of $V$. In [13, Theorem 3.22] (see also [12, Satz (I.6)] for the case $R=\mathbb{Z})$ Plesken has shown that there exist $R$-forms $M_{0}=M, M_{1}, \ldots, M_{t}$ of $V$ such that

(a) $M_{1}$ is the unique maximal $R G$-sublattice of $M_{0}$,

(b) for $i \in\{1, \ldots, t-1\}, M_{i+1} \neq \pi M_{i-1}$ are the only maximal $R G$-sublattices of $M_{i}$

(c) $\pi M_{t-1}$ is the unique maximal $R G$-sublattice of $M_{s}$.

Moreover, $M_{0}, \ldots, M_{t}$ are representatives of the isomorphism classes of $R$-forms of $V$. The lattice of full-rank $R G$-sublattices of $M$ therefore looks as in diagram (9) below.

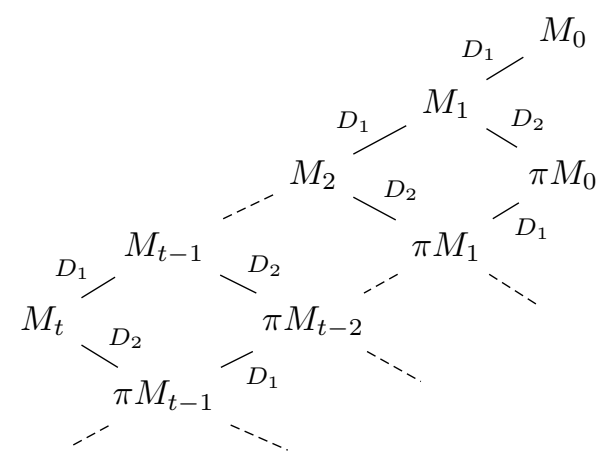

We set $\operatorname{dim}_{k}\left(M_{0} / M_{1}\right)=\operatorname{dim}_{k}\left(D_{1}\right)=: d_{1}$ and $\operatorname{dim}_{k}\left(M_{1} / \pi M_{0}\right)=\operatorname{dim}_{k}\left(D_{2}\right)=: d_{2}$, so that $d=d_{1}+d_{2}$. 
Lemma 4.8. The matrix $\mathbf{A}_{M}=\left(A_{i j}\right) \in \mathbb{Z}[X]^{(t+1) \times(t+1)}$ satisfies

$$
A_{i j}= \begin{cases}1 & \text { if } i=j=1 \text { or } i=j=t+1, \\ 1+X^{d} & \text { if } i=j, i \notin\{1, t+1\} \\ -X^{d_{1}} & \text { if } j=i+1 \\ -X^{d_{2}} & \text { if } j=i-1, \\ 0 & \text { otherwise. }\end{cases}
$$

Thus

$$
A=\left(\begin{array}{cccccc}
1 & -X^{d_{1}} & 0 & 0 & \ldots & 0 \\
-X^{d_{2}} & 1+X^{d} & -X^{d_{1}} & 0 & \ldots & 0 \\
\vdots & \vdots & \vdots & \vdots & \vdots & \vdots \\
0 & 0 & 0 & -X^{d_{2}} & 1+X^{d} & -X^{d_{1}} \\
0 & 0 & 0 & 0 & -X^{d_{2}} & 1
\end{array}\right)
$$

Proof. From 4.7 and diagram (9) we can read off the following properties. First of all we have $\max \left(M_{0}\right)=\left\{M_{1}\right\}, \max \left(M_{t}\right)=\left\{\pi M_{t-1}\right\}$, and $\max \left(M_{i}\right)=\left\{M_{i}, \pi M_{i-1}\right\}$, for $i \in\{1, \ldots, t-1\}$. In particular, $\Phi\left(M_{0}\right)=\left\{M_{0}, M_{1}\right\}, \Phi\left(M_{t}\right)=\left\{M_{t}, \pi M_{t-1}\right\}$ and $\Phi\left(M_{i}\right)=\left\{M_{i}, M_{i+1}, \pi M_{i-1}, \pi M_{i}\right\}$, for $i \in\{1, \ldots, t-1\}$. Since $M_{0}, \ldots, M_{t}$ are representatives of the isomorphism classes of $R$-forms of $V$, we conclude that $\Phi\left(M_{0}, M_{j}\right)=\left\{M_{j}\right\}$, for $j \in\{0,1\}$, and $\Phi\left(M_{0}, M_{j}\right)=\emptyset$ otherwise. Moreover, for $i \in\{1, \ldots, t\}$, we have

$$
\Phi\left(M_{i}, M_{j}\right)= \begin{cases}\left\{M_{i}, \pi M_{i}\right\} & \text { if } i=j, i \neq t, \\ \left\{M_{i}\right\} & \text { if } i=j, i=t, \\ \left\{M_{i+1}\right\} & \text { if } j=i+1, \\ \left\{\pi M_{i-1}\right\} & \text { if } j=i-1 .\end{cases}
$$

From this the assertion of the lemma follows.

Proposition 4.9. The matrix $\mathbf{B}=\left(B_{i j}\right)_{1 \leqslant i, j \leqslant t+1}$ defined by

$$
B_{i j}=\frac{1}{1-X^{d}} \begin{cases}X^{(j-i) d_{1}} & \text { if } j \geqslant i \\ X^{(i-j) d_{2}} & \text { if } i \geqslant j .\end{cases}
$$


satisfies $\mathbf{B}_{M}=\mathbf{B}$. Thus

$$
\mathbf{B}_{M}=\frac{1}{1-X^{d}}\left(\begin{array}{ccccccc}
1 & X^{d_{1}} & X^{2 d_{1}} & X^{3 d_{1}} & X^{4 d_{1}} & \cdots & X^{t d_{1}} \\
X^{d_{2}} & 1 & X^{d_{1}} & X^{2 d_{1}} & X^{3 d_{1}} & \cdots & X^{(t-1) d_{1}} \\
X^{2 d_{2}} & X & 1 & X^{d_{1}} & X^{2 d_{1}} & \cdots & X^{(t-2) d_{1}} \\
\vdots & \vdots & \vdots & \vdots & \vdots & \vdots & \vdots \\
X^{(t-2) d_{2}} & X^{(t-3) d_{2}} & \ldots & X & 1 & X^{d_{1}} & X^{2 d_{1}} \\
X^{(t-1) d_{2}} & X^{(t-2) d_{2}} & X^{(t-3) d_{2}} & \ldots & X & 1 & X^{d_{1}} \\
X^{t d_{2}} & X^{(t-1) d_{2}} & X^{(t-2) d_{2}} & \cdots & X^{2 d_{2}} & X^{d_{2}} & 1
\end{array}\right) .
$$

Proof. As usual we prove the assertion by showing that the matrix $\mathbf{B}$ is the inverse of $\mathbf{A}_{M}$, which is a straightforward calculation. Let $A_{i}$ be the $i$ th row of $\mathbf{A}_{M}$ and $B_{j}$ the $j$ th column of $\mathbf{B}$. We need to show that $A_{i} B_{j}=\delta_{i j}$, for $i, j \in\{1, \ldots, t+1\}$. For instance, for $i, j \in\{2, \ldots, t-1\}$, we have

$$
\begin{aligned}
A_{i} \cdot B_{j} & =A_{i, i-1} B_{j, i-1}+A_{i, i} B_{j, i}+A_{i, i+1} B_{j, i+1} \\
& =-X^{d_{2}} B_{j, i-1}+\left(1+X^{d_{1}+d_{2}}\right) B_{j, i}-X^{d_{1}} B_{j, i+1} .
\end{aligned}
$$

Now $A_{i} B_{j}=\delta_{i j}$ follows by observing that

$$
\left(B_{j, i-1}, B_{j, i}, B_{j, i+1}\right)= \begin{cases}\left(X^{(j-i+1) d_{1}}, X^{\left.(j-i) d_{1}\right)}, X^{(j-i-1) d_{1}}\right) & \text { if } i<j \\ \left(X^{d_{1}}, 1, X^{d_{2}}\right) & \text { if } i=j \\ \left(X^{(i-j-1) d_{2}}, X^{(i-j) d_{2}}, X^{(i-j+1) d_{2}}\right) & \text { if } i>j .\end{cases}
$$

The remaining cases are treated analogously.

We have now established the following:

Corollary 4.10. With the notation as in 4.7, for $i \in\{0, \ldots, t\}$, one has

$$
Z\left(M_{i}\right)=\frac{1}{1-X^{d}}\left(\sum_{j=0}^{i} X^{j d_{2}}+\sum_{j=1}^{t-i} X^{j d_{1}}\right) \text {. }
$$

\subsection{Modular reductions with three non-isomorphic composition factors.}

Throughout this subsection, let $R$ be a local principal ideal domain with maximal ideal $\mathfrak{m}=(\pi)$, field of fractions $K$, and finite residue field $k=R / \mathfrak{m}$ of cardinality q. Assume further that $G$ is a finite group and $S=S_{1}$ is an $R$-form of an absolutely simple $K G$-module $V$ satisfying Hypotheses 3.1 . Let $S_{1}, S_{2}, \ldots, S_{3 t+1}$ be $R G$-lattices as in Theorem 3.2, and suppose that $S_{1}, S_{2}, \ldots, S_{3 t+1}$ are representatives of the isomorphism classes of $R$-forms of $V$.

Our next aim in this subsection is to determine $Z(S)$. 
Lemma 4.11. With the above notation, let

$$
\mathbf{A}_{1}=\left(\begin{array}{cccc}
1 & -X^{d_{2}} & -X^{d_{3}} & X^{d_{2}+d_{3}} \\
0 & 1 & 0 & -X^{d_{3}} \\
0 & 0 & 1 & -X^{d_{2}}
\end{array}\right) \in \mathbb{Z}[X]^{3 \times 4}
$$

Then

$$
\mathbf{A}_{S}=\left[\begin{array}{c|c}
\mathbf{A}_{1} & \mathbf{0}_{3 \times 3(t-1)} \\
\hline *
\end{array}\right]
$$

Proof. From Theorem 3.2 and diagram (7) we know that $\max \left(S_{1}\right)=\left\{S_{2}, S_{3}\right\}$, $\max \left(S_{2}\right)=\max \left(S_{3}\right)=\left\{S_{4}\right\}$, and $\operatorname{Rad}\left(S_{i}\right)=S_{4}$, for $i \in\{1,2,3\}$. Thus $\Phi\left(S_{1}\right)=$ $\left\{S_{1}, S_{2}, S_{3}, S_{4}\right\}$ and $\Phi\left(S_{i}\right)=\left\{S_{i}, S_{4}\right\}$, for $i \in\{2,3\}$. As $S_{1}, \ldots, S_{3 t+1}$ are representatives of the isomorphism classes of $R$-forms of $V$, we obtain $\Phi\left(S_{1}, S_{j}\right)=\left\{S_{j}\right\}$ for $j \in\{1,2,3,4\}$, and $\Phi\left(S_{1}, S_{j}\right)=\emptyset$ otherwise. As well, for $i \in\{2,3\}$, we have $\Phi\left(S_{i}, S_{j}\right)=\left\{S_{j}\right\}$ for $j \in\{i, 4\}$, and $\Phi\left(S_{i}, S_{j}\right)=\emptyset$ otherwise. From this the assertion of the lemma follows.

Lemma 4.12. Let $i \in\{1, \ldots, t-1\}$, and let $\mathbf{A}^{\prime}=\left(A_{l j}\right)_{3 i+1 \leqslant l \leqslant 3 i+3,1 \leqslant j \leqslant 3 t+1}$ be the submatrix of $\mathbf{A}_{S}$ consisting of rows $3 i+1,3 i+2,3 i+3$. Then

$$
\mathbf{A}^{\prime}=\left[\begin{array}{l|l|l}
\mathbf{0}_{3 \times 3(i-1)}\left|\mathbf{A}_{2}\right| \mathbf{0}_{3 \times 3(t-i)-3}
\end{array}\right],
$$

where

$\mathbf{A}_{2}=\left(\begin{array}{ccccccc}-X^{d_{1}} & X^{d_{1}+d_{2}} & X^{d_{1}+d_{3}} & 1-X^{d} & -X^{d_{2}} & -X^{d_{3}} & X^{d_{2}+d_{3}} \\ 0 & -X^{d_{1}} & 0 & X^{d_{1}+d_{3}} & 1 & 0 & -X^{d_{3}} \\ 0 & 0 & -X^{d_{1}} & X^{d_{1}+d_{2}} & 0 & 1 & -X^{d_{2}}\end{array}\right) \in \mathbb{Z}[X]^{3 \times 7}$.

Proof. Let $i \in\{1, \ldots, t-1\}$. We use again Theorem 3.2 and diagram (7) to conclude that $\max \left(S_{3 i+1}\right)=\left\{S_{3 i+2}, S_{3 i+3}, \pi S_{3(i-1)+1}\right\}, \max \left(S_{3 i+2}\right)=\left\{S_{3(i+1)+1}, \pi S_{3(i-1)+2}\right\}$ and $\max \left(S_{3 i+3}\right)=\left\{S_{3(i+1)+1}, \pi S_{3(i-1)+3}\right\}$. In particular, we obtain $\operatorname{Rad}\left(S_{3 i+1}\right)=$ $\operatorname{Rad}\left(S_{3 i+2}\right)=\operatorname{Rad}\left(S_{3 i+3}\right)=\pi S_{3 i+1}$ and

$$
\begin{aligned}
& \Phi\left(S_{3 i+1}\right)=\left\{S_{3 i+1}, S_{3 i+2}, S_{3 i+3}, S_{3(i+1)+1},\right. \\
&\left.\pi S_{3(i-1)+1}, \pi S_{3(i-1)+2}, \pi S_{3(i-1)+3}, \pi S_{3 i+1}\right\}, \\
& \Phi\left(S_{3 i+2}\right)=\left\{S_{3(i+1)+1}, \pi S_{3(i-1)+2}, \pi S_{3 i+1}\right\}, \\
& \Phi\left(S_{3 i+3}\right)=\left\{S_{3(i+1)+1}, \pi S_{3(i-1)+3}, \pi S_{3 i+1}\right\} .
\end{aligned}
$$


Thus

$\Phi\left(S_{3 i+1}, S_{j}\right)= \begin{cases}\left\{S_{j}\right\} & \text { if } j \in\{3 i+2,3 i+3\}, \\ \left\{\pi S_{j}\right\} & \text { if } j \in\{3(i-1)+1,3(i-1)+2,3(i-1)+3\}, \\ \left\{S_{3 i+1} \pi S_{3 i+1}\right\} & \text { if } j=3 i+1, \\ \emptyset & \text { otherwise, }\end{cases}$ $\Phi\left(S_{3 i+2}, S_{j}\right)=\Phi\left(S_{3 i+3}, S_{j}\right)=\left\{S_{j}\right\}$ for $j=3(i+1)+1, \Phi\left(S_{3 i+2}, S_{j}\right)=\left\{\pi S_{j}\right\}$ for $j \in\{3(i-1)+2,3 i+1\}$ and $\Phi\left(S_{3 i+2}, S_{j}\right)=\emptyset$ otherwise. Analogously, $\Phi\left(S_{3 i+3}, S_{j}\right)=$ $\left\{\pi S_{j}\right\}$ for $j \in\{3(i-1)+3,3 i+1\}$, and $\Phi\left(S_{3 i+3}, S_{j}\right)=\emptyset$ otherwise.

We have now determined all the rows of $\mathbf{A}_{S}$, except for the last one.

Lemma 4.13. The last row of $\mathbf{A}_{S}$ is equal to $\left[\mathbf{0}_{1 \times(3 t-6)} \mid \mathbf{A}_{3}\right]$, where

$$
\mathbf{A}_{3}=\left(\begin{array}{lllllll}
0 & 0 & 0 & -X^{d_{1}} & 0 & 0 & 1
\end{array}\right) \in \mathbb{Z}[X]^{1 \times 7} .
$$

Proof. We have $\max \left(S_{3 t+1}\right)=\left\{\pi S_{3(t-1)+1}\right\}$ and $\operatorname{Rad}\left(S_{3 t+1}\right)=\pi S_{3(t-1)+1}$. Hence $\Phi\left(S_{3 t+1}\right)=\left\{S_{3 t+1}, \pi S_{3(t-1)+1}\right\}$, and it follows that $\Phi\left(S_{3 t+1}, S_{j}\right)=\left\{S_{j}\right\}$ for $j=$ $3 t+1, \Phi\left(S_{3 t+1}, S_{j}\right)=\left\{\pi S_{j}\right\}$ for $j=3(t-1)+1$, and $\Phi\left(S_{3 t+1}, S_{j}\right)=\emptyset$ otherwise.

To summarize, we have now established

Proposition 4.14. The matrix $\mathbf{A}_{S}$ is given as follows:

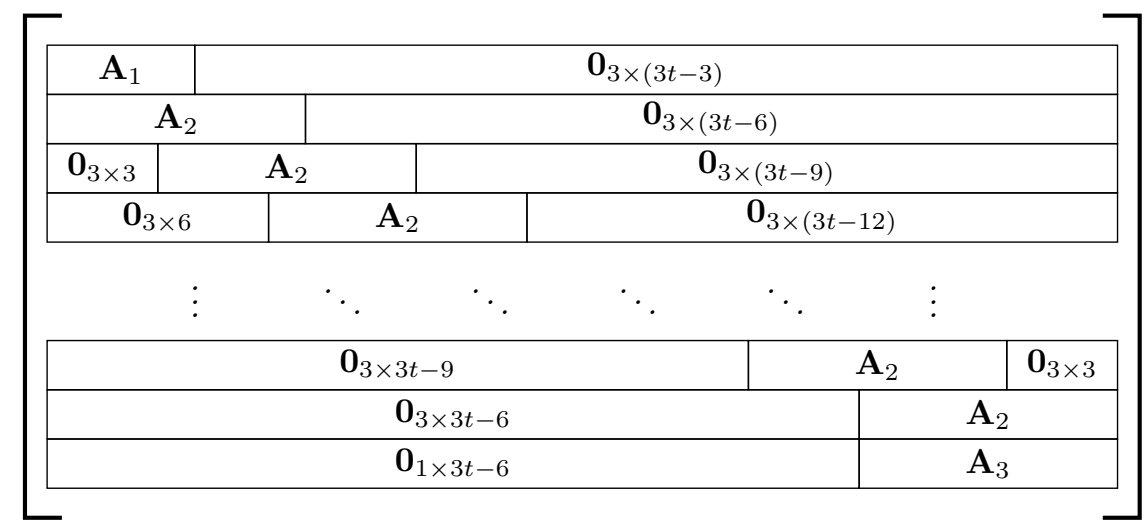

4.15. The matrix B. We shall now define a matrix $\mathbf{B}=\left(B_{i j}\right) \in \mathbb{Q}(X)^{(3 t+1) \times(3 t+1)}$, and shall successively prove that this is precisely the matrix $\mathbf{B}_{S}$. For $i, j \in$ $\{1, \ldots, 3 t+1\}$, we write $B_{i j}=b_{i j} \cdot \frac{1}{1-X^{d}}$, where $b_{i j}$ is given as follows:

(a) If $m \equiv 1(\bmod 3)$, then, for $i \geqslant 0$, we set $b_{m+3 i+1, m}=X^{(i+1) d_{1}+d_{3}}$, $b_{m+3 i+2, m}=X^{(i+1) d_{1}+d_{2}}$, and $b_{m+3 i+3, m}=X^{(i+1) d_{1}}$. 
(b) If $m \equiv 2(\bmod 3)$, then, for $i \geqslant 0$, we set $b_{m+3 i+1, m}=X^{(i+1) d_{1}+2 d_{2}}$, $b_{m+3 i+2, m}=X^{(i+1) d_{1}+d_{2}}$, and $b_{m+3 i+3, m}=X^{(i+1) d_{1}}$.

(c) If $m \equiv 0(\bmod 3)$, then, for $i \geqslant 0$, we set $b_{m+3 i+1, m}=X^{(i+1) d_{1}+d_{3}}$, $b_{m+3 i+2, m}=X^{(i+2) d_{1}+2 d_{3}}$, and $b_{m+3 i+3, m}=X^{(i+1) d_{1}}$.

(d) If $m \equiv 1(\bmod 3)$, then, for $i \geqslant 0$, we set $b_{m, m+3 i+1}=X^{(i+1) d_{2}+i d_{3}}$, $b_{m, m+3 i+2}=X^{i d_{2}+(i+1) d_{3}}$, and $b_{m, m+3 i+3}=X^{(i+1)\left(d_{2}+d_{3}\right)}$.

(e) If $m \equiv 2(\bmod 3)$, then we set $b_{m, m+1}=X^{d_{1}+2 d_{3}}, b_{m, m+3 i+1}=X^{(i-1) d_{2}+(i+1) d_{3}}$ for $i \geqslant 1, b_{m, m+3 i+2}=X^{i d_{2}+(i+1) d_{3}}$ for $i \geqslant 0$, and $b_{m, m+3 i+3}=X^{(i+1)\left(d_{2}+d_{3}\right)}$ for $i \geqslant 0$.

(f) If $m \equiv 0(\bmod 3)$, then, for $i \geqslant 0$, we set $b_{m, m+3 i+1}=X^{(i+1) d_{2}+i d_{3}}$, $b_{m, m+3 i+2}=X^{(i+2) d_{2}+i d_{3}}$, and $b_{m, m+3 i+3}=X^{(i+1)\left(d_{2}+d_{3}\right)}$.

Moreover, we set $b_{i i}:=1$, for $i \geqslant 1$.

We aim to show that $\mathbf{B}$ is the inverse of $\mathbf{A}$. To do this, we partition $\left(1-X^{d}\right) \cdot \mathbf{B}$ into blocks: First we define $\mathbf{B}_{1}=\left(b_{i j}\right)_{1 \leqslant i, j \leqslant 7} \in \mathbb{Z}[X]^{7 \times 7}$, that is,

$\mathbf{B}_{1}=\left(\begin{array}{ccccccc}1 & X^{d_{2}} & X^{d_{3}} & X^{d_{2}+d_{3}} & X^{2 d_{2}+d_{3}} & X^{d_{2}+2 d_{3}} & X^{2 d_{2}+2 d_{3}} \\ X^{d_{1}+d_{3}} & 1 & X^{d_{1}+2 d_{3}} & X^{d_{3}} & X^{d_{2}+d_{3}} & X^{2 d_{3}} & X^{d_{2}+2 d_{3}} \\ X^{d_{1}+d_{2}} & X^{d_{1}+2 d_{2}} & 1 & X^{d_{2}} & X^{2 d_{2}} & X^{d_{2}+d_{3}} & X^{2 d_{2}+d_{3}} \\ X^{d_{1}} & X^{d_{1}+d_{2}} & X^{d_{1}+d_{3}} & 1 & X^{d_{2}} & X^{d_{3}} & X^{d_{2}+d_{3}} \\ X^{2 d_{2}+d_{3}} & X^{d_{1}} & X^{2 d_{1}+2 d_{3}} & X^{d_{1}+d_{3}} & 1 & X^{d_{1}+2 d_{3}} & X^{d_{3}} \\ X^{2 d_{1}+d_{2}} & X^{2 d_{1}+2 d_{2}} & X^{d_{1}} & X^{d_{1}+d_{2}} & X^{d_{1}+2 d_{2}} & 1 & X^{d_{2}} \\ X^{2 d_{1}} & X^{2 d_{1}+d_{2}} & X^{2 d_{1}+d_{3}} & X^{d_{1}} & X^{d_{1}+d_{2}} & X^{d_{1}+d_{3}} & 1\end{array}\right)$.

Next we set $\mathbf{B}_{3}=\left(b_{i j}\right)_{(3 t+1)-7 \leqslant i \leqslant 3 t+1,1 \leqslant j \leqslant 3 t+1} \in \mathbb{Z}[X]^{7 \times(3 t+1-7)}$ and $\mathbf{B}_{2}=$ $\left(b_{i j}\right)_{1 \leqslant i \leqslant 7,8 \leqslant j \leqslant 3 t+1} \in \mathbb{Z}[X]^{7 \times(3 t+1-7)}$. Thus we have

$$
\mathbf{B}=\frac{1}{1-X^{d}}\left(\begin{array}{c|c}
\mathbf{B}_{1} \mid \mathbf{B}_{2} \\
\hline \multirow{2}{*}{*} \\
\hline \mathbf{B}_{3} & \mathbf{B}_{1}
\end{array}\right)
$$

Let $D=\left(1-X^{d}\right)$

Lemma 4.16. With the above notation, the following holds:

(a) For $l \in\{1, \ldots, t-2\}$, one has $\mathbf{B}_{1}=\left(b_{i j}\right)_{\substack{3 l+1 \leqslant i \leqslant 3 l+7, 3 l+1 \leqslant j \leqslant 3 l+7}}$.

(b) For $l \in\{1, \ldots, t-2\}$, there exists some $c_{l}^{\prime} \in \mathbb{Q}(X)$ such that

$$
\left(b_{i j}\right)_{3 l+1 \leqslant i \leqslant 3 l+7},=c_{l}^{\prime} \cdot\left(\left(\mathbf{B}_{3}\right)_{i j}\right)_{\substack{1 \leqslant i \leqslant 7 \\ 1 \leqslant j \leqslant 3 l}} \cdot
$$


(c) For $l \in\{1, \ldots, t-2\}$, there exists some $c_{l}^{\prime \prime} \in \mathbb{Q}(X)$ such that

$$
\left(b_{i j}\right)_{\substack{3 l+1 \leqslant i \leqslant 3 l+7, 3 l+8 \leqslant j \leqslant 3 t+1}}=c_{l}^{\prime \prime} \cdot\left(\left(\mathbf{B}_{2}\right)_{i j}\right)_{\substack{1 \leqslant i \leqslant 7, 3 l+8 \leqslant j \leqslant 3 t+1}} .
$$

Proof. Let $l \in\{1, \ldots, t-2\}$, and let $i, j \in\{1, \ldots, 7\}$. Then 4.15 immediately gives $b_{i+3 l, j+3 l}=b_{i j}$, proving (a).

Next let $m \in\{1, \ldots, 3 t-2\}$ and $r \in\{1, \ldots, m\}$. Then, by $4.15(\mathrm{a})-(\mathrm{c})$, we have $b_{m, r}=X^{-d_{1}} \cdot b_{m+3, r}$. This, in particular, implies (b).

If $r \in\{m+4, \ldots, 3 t-1\}$ and $r>m+4$ in the case where $m \equiv 2(\bmod 3)$, then $4.15(\mathrm{~d})-(\mathrm{f})$ gives $b_{m, r}=X^{d_{2}+d_{3}} \cdot b_{m+3, r}$, which implies (c).

Lemma 4.17. With the above notation, one has the following:
(a) $\mathbf{A}_{2} \cdot \mathbf{B}_{1}=\left[\mathbf{0}_{3 \times 3}\left|D \cdot \mathbf{1}_{3}\right| \mathbf{0}_{3 \times 1}\right]$,
(b) $\mathbf{A}_{2} \cdot \mathbf{B}_{2}=\mathbf{0}$,
(c) $\mathbf{A}_{2} \cdot \mathbf{B}_{3}=\mathbf{0}$,
(d) $\left[\mathbf{A}_{1} \mid \mathbf{0}_{3 \times 3}\right] \cdot \mathbf{B}_{1}=\left[D \cdot \mathbf{1}_{3} \mid \mathbf{0}_{3 \times 4}\right]$,
(e) $\left[\mathbf{0}_{1 \times(3 t-6)} \mid \mathbf{A}_{3}\right] \cdot \mathbf{B}=\left[\mathbf{0}_{1 \times 3 t} \mid D \cdot \mathbf{1}_{1}\right]$,
(f) $\left[\mathbf{A}_{1} \mid \mathbf{0}_{3 \times 3}\right] \cdot \mathbf{B}_{2}=\mathbf{0}$.

Proof. To prove (a) and (d), we compute

$$
\left(\begin{array}{c|c}
\mathbf{A}_{1} & \mathbf{0} \\
\hline \mathbf{A}_{2}
\end{array}\right) \cdot \mathbf{B}_{1}=\left[D \cdot \mathbf{1}_{6} \mid \mathbf{0}_{6 \times 1}\right] .
$$

Next we show (b) and (f): We claim that every column of $\mathbf{B}_{2}$ is of the form

$$
a \cdot\left(1, X^{-d_{2}}, X^{-d_{3}}, X^{-d_{2}-d_{3}}, X^{-2 d_{2}-d_{3}}, X^{-d_{2}-2 d_{3}}, X^{-2 d_{2}-2 d_{3}}\right)^{t},
$$

for some $a \in \mathbb{Q}(X)$. Once we have this, we immediately get

$$
\left[\begin{array}{c|c}
\mathbf{A}_{1} & \mathbf{0}_{3 \times 3} \\
\hline \mathbf{A}_{2}
\end{array}\right] \cdot \mathbf{B}_{2}=0 .
$$

Obviously, (10) is true for the first three columns of $\mathbf{B}_{2}$. So suppose that $m \in$ $\{1, \ldots, 7\}$ and $r \in\{8, \ldots, 3 t-2\}$. Then $4.15(\mathrm{~d})$-(f) shows that $b_{m, r+3}=X^{d_{2}+d_{3}}$. $b_{m, r}$, whence $(10)$.

To prove (c), we first claim that, for $i \in\{1, \ldots, 3 t-6\}$, the $i$ th column of $\mathbf{B}_{3}$ has the form

$$
\begin{array}{rcc}
a_{i}\left(X^{-2 d_{1}}, X^{d_{3}-d_{1}}, X^{d_{2}-d_{1}}, X^{-d_{1}}, X^{d_{3}}, X^{d_{2}}, 1\right)^{t} & \text { if } i \equiv 1 & (\bmod 3), \\
a_{i}\left(X^{-2 d_{1}}, X^{-d_{2}-2 d_{1}}, X^{d_{2}-d_{1}}, X^{-d_{1}}, X^{-d_{2}-d_{1}}, X^{d_{2}}, 1\right)^{t} & \text { if } i \equiv 2 & (\bmod 3), \\
a_{i}\left(X^{-2 d_{1}}, X^{-d_{1}+d_{3}}, X^{-2 d_{1}-d_{3}}, X^{-d_{1}}, X^{d_{3}}, X^{-d_{1}-d_{3}}, 1\right)^{t} & \text { if } i \equiv 0 & (\bmod 3),
\end{array}
$$


for suitable $a_{i} \in \mathbb{Q}(X)$. Once we have this, we immediately get $\mathbf{A}_{2} \cdot \mathbf{B}_{3}=\mathbf{0}$. The claim is easily verified for the first three columns of $\mathbf{B}_{3}$. If $m \in\{3 t-5, \ldots, 3 t+1\}$ and $r \in\{1, \ldots, 3 t-9\}$, then $4.15(\mathrm{a})-(\mathrm{c})$ gives $b_{m, r}=X^{d_{1}} \cdot b_{m, r+3}$. Thus the claim follows by induction.

It remains to prove (e). To do so, it is sufficient to compute

$$
\mathbf{A}_{3} \cdot\left[\mathbf{B}_{3} \mid \mathbf{B}_{1}\right]
$$

Denoting by $\mathbf{B}_{3, i}$ the $i$ th column of $\mathbf{B}_{3}$, we have

$$
\mathbf{A}_{3} \cdot \mathbf{B}_{3, i}=\left(1-X^{d}\left(a_{i}-a_{i}\right)\right)=0 .
$$

On the other hand, a quick calculation shows

$$
\mathbf{A}_{3} \cdot \mathbf{B}_{1}=\left(\begin{array}{lllllll}
0 & 0 & 0 & 0 & 0 & 0 & 1-X^{d}
\end{array}\right)
$$

which finishes the proof.

We now obtain

Theorem 4.18. The matrix $\mathbf{B}$ defined in 4.15 is the inverse of $\mathbf{A}_{S}$, that is, $\mathbf{B}=$ $\mathbf{B}_{S}$.

Proof. We show that $\mathbf{A}_{S} \cdot \mathbf{B}=\mathbf{1}_{3 t+1}$. To this end, we multiply $\mathbf{B}$ with the rows of A successively from the left. First note that, by Lemma 4.17, we have

$$
\left[\begin{array}{c|c|c}
\mathbf{A}_{1} & \mathbf{0}_{3 \times 3} & \mathbf{0}_{3 \times 3 t-6} \\
\hline \mathbf{A}_{2} & \mathbf{0}_{3 \times 3 t-6}
\end{array}\right] \cdot \mathbf{B}=\left[\begin{array}{c|c}
\mathbf{A}_{1} \mid \mathbf{0}_{3 \times 3} \\
\hline \mathbf{A}_{2}
\end{array}\right] \cdot\left[\mathbf{B}_{1} \mid \mathbf{B}_{2}\right]=\left[\mathbf{1}_{6} \mid \mathbf{0}_{6 \times 3 t-5}\right] .
$$

Next consider

$$
\left[\mathbf{0}_{3 \times 3}\left|\mathbf{A}_{2}\right| \mathbf{0}_{3 t-9 \times 3 t-9}\right] \cdot \mathbf{B}=\mathbf{A}_{2} \cdot\left(B_{i j}\right)_{4 \leqslant i \leqslant 10,1 \leqslant j \leqslant 3 t+1} .
$$

Let us write $\left(B_{i j}\right)_{4 \leqslant i \leqslant 10,1 \leqslant j \leqslant 3 t+1}=\left[\mathbf{B}^{\prime}\left|\mathbf{B}_{1}\right| \mathbf{B}^{\prime \prime}\right]$ with $\mathbf{B}^{\prime}=\left(B_{i j}\right)_{4 \leqslant i \leqslant 10,1 \leqslant j \leqslant 3}$ and $\mathbf{B}^{\prime \prime}=\left(B_{i j}\right)_{4 \leqslant i \leqslant 10,11 \leqslant j \leqslant 3 t+1}$. By Lemma 4.16, we know $\mathbf{B}^{\prime}=c^{\prime}\left(\left(\mathbf{B}_{3}\right)_{i j}\right)_{1 \leqslant i \leqslant 7,1 \leqslant j \leqslant 3}$ and $\mathbf{B}^{\prime \prime}=c^{\prime \prime}\left(\left(\mathbf{B}_{2}\right)_{i j}\right)_{1 \leqslant i \leqslant 7,11 \leqslant j \leqslant 3 t+1}$, for some $c^{\prime}, c^{\prime \prime} \in \mathbb{Q}(X)$. Together with Lemma 4.17 this shows that

$$
\begin{aligned}
{\left[\mathbf{0}_{3 \times 3}\left|\mathbf{A}_{2}\right| \mathbf{0}_{3 \times 3 t-9}\right] \cdot \mathbf{B}=\mathbf{A}_{2} \cdot\left[\mathbf{B}^{\prime}\left|\mathbf{B}_{1}\right| \mathbf{B}^{\prime \prime}\right] } & =\left[\mathbf{A}_{2} \cdot \mathbf{B}^{\prime}\left|\mathbf{A}_{2} \cdot \mathbf{B}_{1}\right| \mathbf{A}_{2} \cdot \mathbf{B}^{\prime \prime}\right] \\
& =\left[\mathbf{0}_{3 \times 6}\left|\mathbf{1}_{3}\right| \mathbf{0}_{3 \times 3 t-8}\right]
\end{aligned}
$$


We now do the previous step for an arbitrary slice of $\mathbf{A}_{S}$. Let $1 \leqslant l \leqslant t-1$ and consider

$$
\begin{aligned}
\left(A_{i j}\right)_{\substack{3 l+1 \leqslant i \leqslant 3(l+1), 1 \leqslant j \leqslant 3 t+1}} \cdot \mathbf{B} & =\left[\mathbf{0}_{3 \times 3(l-1)}\left|\mathbf{A}_{2}\right| \mathbf{0}_{3 \times 3(t-l-1)}\right] \cdot \mathbf{B} \\
& =\mathbf{A}_{2} \cdot\left(B_{i j}\right)_{3(l-1)+1 \leqslant i \leqslant 3(l-1)+7,}, \\
1 \leqslant j \leqslant 3 t+1 &
\end{aligned}
$$

We write

$$
\begin{aligned}
\mathbf{B}^{\prime} & =\left(B_{i j}\right)_{3(l-1)+1 \leqslant i \leqslant 3(l-1)+7,1 \leqslant j \leqslant 3(l-1)}, \\
\mathbf{B}^{\prime \prime} & =\left(B_{i j}\right)_{3(l-1)+1 \leqslant i \leqslant 3(l-1)+7,3(l-1)+8 \leqslant j \leqslant 3 t+1},
\end{aligned}
$$

and note that $\mathbf{B}_{1}=\left(B_{i j}\right)_{3(l-1)+1 \leqslant i \leqslant 3(l-1)+7,3(l-1)+1 \leqslant j \leqslant 3(l-1)+7}$. By Lemma 4.16, we know $\mathbf{B}^{\prime}=c^{\prime}\left(\left(\mathbf{B}_{3}\right)_{i j}\right)_{1 \leqslant i \leqslant 7,1 \leqslant j \leqslant 3(l-1)}$ and $\mathbf{B}^{\prime \prime}=c^{\prime \prime}\left(\left(\mathbf{B}_{2}\right)_{i j}\right)_{1 \leqslant i \leqslant 7,3(l-1)+8 \leqslant j \leqslant 3 t+1}$, for some $c^{\prime}, c^{\prime \prime} \in \mathbb{Q}(X)$. Thus, using Lemma 4.17,

$$
\begin{aligned}
\left(A_{i j}\right)_{\substack{3 l+1 \leqslant i \leqslant 3(l+1) \\
1 \leqslant j \leqslant 3 t+1}} \cdot \mathbf{B} & =\left[\mathbf{A}_{2} \cdot \mathbf{B}^{\prime}\left|\mathbf{A}_{2} \cdot \mathbf{B}_{1}\right| \mathbf{A}_{2} \cdot \mathbf{B}^{\prime \prime}\right] \cdot \mathbf{B} \\
& =\left[\mathbf{0}_{3 \times 3(l-1)}\left|\mathbf{1}_{3}\right| \mathbf{0}_{3 \times 3 t-3(l-1)-3}\right] .
\end{aligned}
$$

Finally note that $\left[\mathbf{0}_{1 \times 3 t-6} \mid \mathbf{A}_{3}\right] \cdot \mathbf{B}=\left[\mathbf{0}_{1 \times 3 t} \mid D \cdot \mathbf{1}_{1}\right]$ by Lemma 4.17

As an immediate consequence of Theorem 4.18 and 4.2 , we have

Corollary 4.19. With the above notation, one has

$$
Z(S)=\frac{1}{1-X^{d}}\left(1+\sum_{i=1}^{t} X^{i d_{2}+i d_{3}}+X^{(i-1) d_{2}+i d_{3}}+X^{i d_{2}+(i-1) d_{3}}\right) .
$$

Remark 4.20. Of course, given $\mathbf{B}_{S}$, we can also read off $Z\left(S_{2}\right), \ldots, Z\left(S_{3 t+1}\right)$. Since the formulae become more complicated and we shall not need them for our applications below, we do, however, not write them down explicitly here.

4.5. Application I: Specht lattices labelled by hook partitions. We want to use the previous results to determine the zeta functions of certain lattices over $p$-adic group algebras, the first of which will come from the symmetric group $\mathfrak{S}_{n}$ of degree $n \geqslant 4$. In the language of 3.4 , our aim is to determine the zeta functions of the Specht lattices associated to the hook partitions $\left(n-r, 1^{r}\right)$, for $r \in\{0, \ldots, n-1\}$. For $r=1$, the zeta functions of $S_{\mathbb{Z}}^{\left(2,1^{n-2}\right)}$ and $S_{\mathbb{Z}_{p}}^{\left(2,1^{n-2}\right)}$, where $p$ is any prime, have been determined by the second author in [6]. Here we shall investigate $S_{\mathbb{Z}_{p}}^{\left(n-r, 1^{r}\right)}$, for all $r \in\{1, \ldots, n-2\}$ and odd primes $p$, as well as $S_{\mathbb{Z}_{2}}^{\left(n-2,1^{2}\right)}$ and $S_{\mathbb{Z}}^{\left(n-2,1^{2}\right)}$. We begin with the local zeta functions at the odd primes $p$. Recall from 3.4 and 3.5 that $S_{\mathbb{Z}_{p}}^{\left(n-r, 1^{r}\right)} \cong \mathbb{Z}_{p} \otimes_{\mathbb{Z}} S_{\mathbb{Z}}^{\left(n-r, 1^{r}\right)}$ and that $\operatorname{rk}_{\mathbb{Z}_{p}}\left(S_{\mathbb{Z}_{p}}^{\left(n-r, 1^{r}\right)}\right)=\operatorname{rk}_{\mathbb{Z}}\left(S_{\mathbb{Z}}^{\left(n-r, 1^{r}\right)}\right)=\left(\begin{array}{c}n-1 \\ r\end{array}\right)$, for $r \in\{0, \ldots, n-1\}$. 
Proposition 4.21. Let $p \geqslant 3$ be a prime number, let $r \in\{1, \ldots, n-2\}$, and let $d:=\left(\begin{array}{c}n-1 \\ r\end{array}\right)$.

(a) If $p \nmid n$, then

$$
\zeta_{\mathbb{Z}_{p} \mathfrak{S}_{n}}\left(S_{\mathbb{Z}_{p}}^{\left(n-r, 1^{r}\right)}, s\right)=\frac{1}{1-p^{-d s}}
$$

(b) If $p \mid n$, then

$$
\zeta_{\mathbb{Z}_{p} \mathfrak{S}_{n}}\left(S_{\mathbb{Z}_{p}}^{\left(n-r, 1^{r}\right)}, s\right)=\frac{1}{1-p^{-d s}} \sum_{i=0}^{\nu_{p}(n)} p^{-s i a}
$$

where $a=\left(\begin{array}{c}n-2 \\ r\end{array}\right)$.

Proof. (a) Since $p \geqslant 3$ is not dividing $n$, by [8, Theorem 23.7], the reduction of $S_{\mathbb{Z}_{p}}^{\left(n-r, 1^{r}\right)}$ modulo $p$ is absolutely simple. So the assertion follows from 4.3.

(b) By $\left[5\right.$, Section 6] we know that there are $\nu_{p}(n)+1$ isomorphism classes of $\mathbb{Z}_{p}$-forms in $S_{\mathbb{Q}_{p}}^{\left(n-r, 1^{r}\right)}$. Moreover the reduction of $S_{\mathbb{Z}_{p}}^{\left(n-r, 1^{r}\right)}$ modulo $p$ has precisely two non-isomorphic composition factors, and is indecomposable. By [8, Theorem 24.1], the head of $S_{\mathbb{Z}_{p}}^{\left(n-r, 1^{r}\right)} / p S_{\mathbb{Z}_{p}}^{\left(n-r, 1^{r}\right)}$ has $\mathbb{F}_{p^{-}}$dimension $d-\left(\begin{array}{c}n-2 \\ r-1\end{array}\right)=\left(\begin{array}{c}n-2 \\ r\end{array}\right)=: a$. Thus, by Corollary 4.10, it follows that

$$
Z\left(S_{\mathbb{Z}_{p}}^{\left(n-r, 1^{r}\right)}\right)=\frac{1}{1-X^{d}} \sum_{i=0}^{\nu_{p}(n)} X^{i a} .
$$

Now the assertion follows from 4.2 .

Remark 4.22. For $r=2$, the next proposition yields the local zeta functions for $p=2$. This will involve the dimension of the simple $\mathbb{F}_{2} \mathfrak{S}_{n}$-module $D_{\mathbb{F}_{2}}^{(n-2,2)}$, for $n>4$, which is well known, by [8, Theorem 24.15]. For $n>4$, one has

$$
\operatorname{dim}_{\mathbb{F}_{2}}\left(D_{\mathbb{F}_{2}}^{(n-2,2)}\right)=\left\{\begin{array}{lll}
\frac{1}{2}\left(n^{2}-5 n+4\right) & \text { if } n \equiv 0 & (\bmod 4), \\
\frac{1}{2}\left(n^{2}-3 n-2\right) & \text { if } n \equiv 1 \quad(\bmod 4), \\
\frac{1}{2}\left(n^{2}-5 n+2\right) & \text { if } n \equiv 2 \quad(\bmod 4), \\
\frac{1}{2}\left(n^{2}-3 n\right) & \text { if } n \equiv 3 \quad(\bmod 4) .
\end{array}\right.
$$

This can also be read off from $[5$, Proposition 7.2$]$, recalling that $\operatorname{dim}_{\mathbb{F}_{2}}\left(D_{\mathbb{F}_{2}}^{(n-1,1)}\right)=$ $n-1$ if $2 \nmid n$, and $\operatorname{dim}_{\mathbb{F}_{2}}\left(D_{\mathbb{F}_{2}}^{(n-1,1)}\right)=n-2$ if $2 \mid n$.

Proposition 4.23. Let $d:=\left(\begin{array}{c}n-1 \\ 2\end{array}\right)$, and let $d_{3}:=\operatorname{dim}_{\mathbb{F}_{2}}\left(D_{\mathbb{F}_{2}}^{(n-2,2)}\right)$ if $n>4$. Then the following hold:

(a) If $n \equiv 1(\bmod 4)$, then

$$
\zeta_{\mathbb{Z}_{2} \mathfrak{S}_{n}}\left(S_{\mathbb{Z}_{2}}^{\left(n-2,1^{2}\right)}, s\right)=\frac{1}{1-2^{-s d}}\left(1+2^{-s}+2^{-s\left(d_{3}+1\right)}\right) .
$$


(b) If $n \equiv 2(\bmod 4)$, then

$$
\zeta_{\mathbb{Z}_{2} \mathfrak{S}_{n}}\left(S_{\mathbb{Z}_{2}}^{\left(n-2,1^{2}\right)}, s\right)=\frac{1}{1-2^{-s d}}\left(1+2^{-s}+2^{-s\left(d_{3}+1\right)}+2^{-s\left(d_{3}+2\right)}\right) .
$$

(c) If $n \equiv 3(\bmod 4)$, then

$$
\zeta_{\mathbb{Z}_{2} \mathfrak{S}_{n}}\left(S_{\mathbb{Z}_{2}}^{\left(n-2,1^{2}\right)}, s\right)=\frac{1}{1-2^{-s d}}\left(1+2^{-s}+2^{-s d_{3}}\right) .
$$

(d) If $n \equiv 0(\bmod 4)$ and $n>4$, then

$$
\begin{aligned}
& \zeta_{\mathbb{Z}_{2} \mathfrak{S}_{n}}\left(S_{\mathbb{Z}_{2}}^{\left(n-2,1^{2}\right)}, s\right)= \\
& \frac{1}{1-2^{-s d}}\left(1+\sum_{i=1}^{\nu_{2}(n)} 2^{-i s-i s d_{3}}+2^{-s(i-1)-s i d_{3}}+2^{-s i-s(i-1) d_{3}}\right) .
\end{aligned}
$$

(e) If $n=4$, then

$$
\zeta_{\mathbb{Z}_{2} \mathfrak{S}_{4}}\left(S_{\mathbb{Z}_{2}}^{\left(2,1^{2}\right)}, s\right)=\frac{1}{1-2^{-s d}}\left(1+2^{-s}+2^{-2 s}\right) .
$$

Proof. We set $M_{1}=S_{\mathbb{Z}_{2}}^{\left(n-2,1^{2}\right)}$. We shall determine $Z\left(M_{1}\right)$, and then apply 4.2 .

(a) Recall that, by [5, Section 7], the (partial) submodule lattice of $M_{1}$ is given by

$$
2 M_{1} \subseteq M_{3} \subseteq M_{2} \subseteq M_{1}
$$

with $M_{1} / M_{2} \cong \mathbb{F}_{2}, M_{2} / M_{3} \cong D_{\mathbb{F}_{2}}^{(n-2,2)}$ and $M_{3} / 2 M_{1} \cong \mathbb{F}_{2}$. Here $\mathbb{F}_{2}$ denotes the trivial $\mathbb{F}_{2} S_{n}$-module. Moreover, $M_{1}, M_{2}, M_{3}$ are representatives of the isomorphism classes of $\mathbb{Z}_{2}$-forms of $S_{\mathbb{Q}_{2}}^{\left(n-1,1^{2}\right)}$. By [5, proof of Theorem 7.10], the reduction modulo 2 of every $\mathbb{Z}_{2}$-form of $S_{\mathbb{Q}_{2}}^{\left(n-2,1^{2}\right)}$ is a uniserial $\mathbb{F}_{2} \mathfrak{S}_{n}$-module. So, by [5, Proposition 3.7 , we can apply Corollary 4.6 , which shows that

$$
Z\left(M_{1}\right)=\frac{1}{1-X^{d}}\left(1+X+X^{d_{3}+1}\right) .
$$

(b) By [5, Section 7], the (partial) submodule lattice of $M_{1}$ is give by

$$
2 M_{1} \subseteq M_{4} \subseteq M_{3} \subseteq M_{2} \subseteq M_{1}
$$

with $M_{1} / M_{2} \cong M_{3} / M_{4} \cong \mathbb{F}_{2}, M_{2} / M_{3} \cong D_{\mathbb{F}_{2}}^{(n-2,2)}$ and $M_{4} / 2 M_{1} \cong D_{\mathbb{F}_{2}}^{(n-1,1)}$. Moreover, $M_{1}, \ldots, M_{4}$ are representatives of the isomorphism classes of $\mathbb{Z}_{2}$-forms of $S_{\mathbb{Q}_{2}}^{\left(n-1,1^{2}\right)}$ In [5, proof of Theorem 7.16] it is also shown that the reduction modulo 2 of every $\mathbb{Z}_{2}$-form of $S_{\mathbb{Q}_{2}}^{\left(n-2,1^{2}\right)}$ is a uniserial $\mathbb{F}_{2} \mathfrak{S}_{n}$-module. Thus, by [5, Proposition 3.7] and Corollary 4.6, we have

$$
Z\left(M_{1}\right)=\frac{1}{1-X^{d}}\left(1+X+X^{d_{3}+1}+X^{d_{3}+2}\right) .
$$


(c) By [5, Proposition 7.9, Theorem 7.10], there are precisely three isomorphism classes of $\mathbb{Z}_{2}$-forms of $S_{\mathbb{Q}_{2}}^{\left(n-2,1^{2}\right)}$. There are representatives $M_{1}, M_{2}, M_{3}$ of these isomorphism classes with the following properties: $M_{1}=S_{\mathbb{Z}_{2}}^{\left(n-2,1^{2}\right)}, M_{2}$ and $M_{3}$ are maximal in $M_{1}, M_{2} \cap M_{3}=2 M_{1}, M_{1} / M_{2} \cong M_{3} / 2 M_{1} \cong \mathbb{F}_{2}, M_{1} / M_{3} \cong M_{2} / 2 M_{1} \cong$ $D_{\mathbb{F}_{2}}^{(n-2,2)}$. Both $M_{2} / 2 M_{2}$ and $M_{3} / 2 M_{3}$ are indecomposable, with head isomorphic to $D_{\mathbb{F}_{2}}^{(n-2,2)}$ and $\mathbb{F}_{2}$, respectively, Thus, we can apply Corollary 4.10 to $M_{2}$ and the chain of sublattices $2 M_{3} \subseteq 2 M_{1} \subseteq M_{2}$. With respect to this ordering, the second row of the matrix $\mathbf{B}_{M_{2}}$ gives

$$
Z\left(M_{1}\right)=\frac{1}{1-X^{d}}\left(1+X+X^{d_{3}}\right) .
$$

(d) This follows from Corollary 4.19 together with Lemma 3.6.

(e) Lastly, let $n=4$ and let $M:=S_{\mathbb{Z}_{2}}^{\left(2,1^{2}\right)}$. Then, by [8, Theorem 8.15], $M / 2 M \cong$ $\left(S_{\mathbb{F}_{2}}^{(3,1)}\right)^{*}$ is indecomposable, with two composition factors, socle isomorphic to $D_{\mathbb{F}_{2}}^{(3,1)}$ and trivial head. So the assertion follows from Corollary 4.10.

Theorem 4.24. Let $n \geqslant 4$, and let $S=S_{\mathbb{Z}}^{\left(n-2,1^{2}\right)}$ be the Specht $\mathbb{Z} \mathfrak{S}_{n}$-lattice labelled by the hook partition $\left(n-2,1^{2}\right)$. Set $d=(n-1)(n-2) / 2$, and $d_{3}=\operatorname{dim}_{\mathbb{F}_{2}}\left(D_{\mathbb{F}_{2}}^{(n-2,2)}\right)$ if $n>4$. Then one has

$$
\zeta_{\mathbb{Z} \mathfrak{S}_{n}}(S, s)=\zeta_{\mathbb{Q}}(d s) \prod_{p \mid n} \varphi_{p}\left(p^{-s}\right),
$$

where $\zeta_{\mathbb{Q}}$ is the Riemann zeta function, $\varphi_{p}(X)=\sum_{i=0}^{\nu_{p}(n)} X^{i} \in \mathbb{Z}[X]$ if $p \geqslant 3$, and

$$
\varphi_{2}(X)=\left\{\begin{array}{lc}
1+X+X^{d_{3}+1} & \text { if } n \equiv 1 \quad(\bmod 4), \\
1+X+X^{d_{3}+1}+X^{d_{3}+2} & \text { if } n \equiv 2 \quad(\bmod 4), \\
1+X+X^{d_{3}} & \text { if } n \equiv 3 \quad(\bmod 4), \\
1+\sum_{i=1}^{\nu_{2}(n)} X^{i+i d_{3}}+X^{(i-1)+i d_{3}}+X^{i+(i-1) d_{3}} & \text { if } n \equiv 0 \quad(\bmod 4), \\
1+X+X^{2} & \text { and } n>4, \\
& \text { if } n=4 .
\end{array}\right.
$$

Proof. Since $V=S_{\mathbb{Q}}^{\left(n-2,1^{2}\right)}$ is an absolutely simple $\mathbb{Q} G$-module of dimension $d$, we have $\zeta_{V}(s)=\zeta_{\mathbb{Q}}(d s)$, as mentioned in 4.3. The claim now follows from 4.3, Proposition 4.21 and Proposition 4.23.

4.6. Application II: projective special linear groups. For the rest of this subsection, let $q$ be a prime power, and let $H=\operatorname{PSL}_{2}(q)$. If $R$ is a principal ideal domain, we denote by $M_{R}$ the permutation $R H$-lattice associated to the action of $H$ on the one-dimensional subspaces of $\mathbb{F}_{q}^{2}$, as defined in 3.13. Let $L_{R} \subseteq M_{R}$ be the $R H$-lattice investigated in 3.10 and 3.13. Our aim is to determine the zeta function 
$\zeta_{\mathbb{Z} H}\left(L_{\mathbb{Z}}, s\right)$. To this end we first compute the local zeta functions $\zeta_{\mathbb{Z}_{p} H}\left(L_{\mathbb{Z}_{p}}, s\right)$, for all primes $p$.

If $R=\mathbb{Z}$ and $K=\mathbb{Q}$, or $R=\mathbb{Z}_{p}$ and $K=\mathbb{Q}_{p}$, for some prime $p$, then we denote by $V_{K}$ the absolutely simple $K H$-module with $R$-form $L_{R}$ as defined in 3.13(b).

Lemma 4.25. Let $p$ be a prime.

(a) If $p \mid q$, or if $p$ is odd with $p \nmid|H|$ or $p \mid(q-1)$, then

$$
\zeta_{\mathbb{Z}_{p} H}\left(L_{\mathbb{Z}_{p}}, s\right)=\frac{1}{1-p^{-q s}} .
$$

(b) If $p$ is odd and $p \mid(q+1)$, then

$$
\zeta_{\mathbb{Z}_{p} H}\left(L_{\mathbb{Z}_{p}}, s\right)=\frac{1}{1-p^{-q s}} \sum_{i=0}^{v_{p}(q+1)} p^{-i(q-1) s} .
$$

Proof. (a) We have already seen in Remark 3.14 that in all three cases $L_{\mathbb{Z}_{p}}$ is up to isomorphism the unique $\mathbb{Z}_{p}$-form of $V_{\mathbb{Q}_{p}}$. Thus the claim follows from 4.2.

(b) By Remark 3.14, we know that $L_{\mathbb{Z}_{p}} / p L_{\mathbb{Z}_{p}}$ has two non-isomorphic composition factors, is indecomposable and has a trivial submodule. Thus, by 3.10 and Corollary 4.10, it follows that

$$
Z\left(L_{\mathbb{Z}_{p}}\right)=\frac{1}{1-X^{q}} \sum_{i=0}^{v_{p}(q+1)} X^{i(q-1)} .
$$

Now the assertion follows from 4.2 .

Lemma 4.26. (a) If $q \equiv \pm 3 \bmod 8$, then

$$
\zeta_{\mathbb{Z}_{2} H}\left(L_{\mathbb{Z}_{2}}, s\right)=\frac{1}{1-2^{-s q}} \sum_{i=0}^{v_{2}(q+1)} 2^{-s i(q-1)} .
$$

(b) If $q \equiv \pm 1 \bmod 8$, then

$$
\zeta_{\mathbb{Z}_{2} H}\left(L_{\mathbb{Z}_{2}}, s\right)=\frac{1}{1-2^{-s q}}\left(1+\sum_{i=1}^{\nu_{2}(q+1)} 2^{-s i(q-1)}+2^{-s(2 i-1)(q-1) / 2+1}\right) .
$$

Proof. (a) This follows from Remark 3.14 (b) and Corollary 4.10.

(b) Lemma 3.17 and Proposition 3.18 show that $L_{\mathbb{Z}_{2}} / 2 L_{\mathbb{Z}_{2}}$ has three composition factors of dimensions $d_{1}=1$ and $d_{2}=d_{3}=(q-1) / 2$, respectively. Moreover, Corollary 4.19 applies, with $t=\nu_{2}(q+1)$. So we get

$$
Z\left(L_{\mathbb{Z}_{2}}\right)=\frac{1}{1-X^{q}}\left(1+\sum_{i=1}^{\nu_{2}(q+1)} X^{i(q-1)}+2 X^{(2 i-1)(q-1) / 2}\right) .
$$

The assertion of the lemma now follows from 4.2. 
Theorem 4.27. The zeta function of the $\mathbb{Z} H$-lattice $L_{\mathbb{Z}}$ is given as

$$
\zeta_{\mathbb{Z} H}\left(L_{\mathbb{Z}}, s\right)=\zeta_{\mathbb{Q}}(q s) \varphi_{2}\left(q^{-s}\right) \prod_{\substack{p \geqslant 3 \\ p \mid q+1}} \varphi_{p}\left(q^{-s}\right),
$$

where $\zeta_{\mathbb{Q}}$ is the Riemann zeta function, $\varphi_{p}(X)=\sum_{i=0}^{\nu_{p}(q+1)} X^{i(q-1)}$ if $p \geqslant 3$ and $p \mid q+1$, and

$$
\varphi_{2}(X)= \begin{cases}\sum_{i=0}^{\nu_{2}(q+1)} X^{i(q-1)} & \text { if } q \equiv \pm 3 \quad(\bmod 8), \\ 1+\sum_{i=1}^{\nu_{2}(q+1)}\left(X^{q-1}+2 X^{(2 i-1)(q-1) / 2}\right) & \text { if } q \equiv \pm 1 \quad(\bmod 8) .\end{cases}
$$

Proof. First note that, since $V_{\mathbb{Q}}$ is an absolutely simple $\mathbb{Q} H$-module of dimension $q$, we have $\zeta_{V}(s)=\zeta_{\mathbb{Q}}(q s)$, as mentioned in 4.3. The assertion of the theorem follows from Lemma 4.25 and Lemma 4.26 together with 4.2 and 4.3.

\section{References}

[1] C. Bonnafé, Representations of $\mathrm{SL}_{2}\left(\mathbb{F}_{q}\right)$, Algebra and Applications, 13, Springer-Verlag London, Ltd., London, 2011.

[2] C. J. Bushnell and I. Reiner, Solomon's conjectures and the local functional equation for zeta functions of orders, Bull. Amer. Math. Soc. (N.S.), 2(2) (1980), 306-310.

[3] M. Craig, A characterization of certain extreme forms, Illinois J. Math., 20(4) (1976), 706-717.

[4] C. W. Curtis and I. Reiner, Methods of Representation Theory, Vol. I. With applications to finite groups and orders, Pure and Applied Mathematics, A Wiley-Interscience Publication, John Wiley \& Sons, Inc., New York, 1981.

[5] S. Danz and T. Hofmann, On integral forms of Specht modules labelled by hook partitions, Preprint, arXiv:1706.02860v2, (2018).

[6] T. Hofmann, Zeta functions of lattices of the symmetric group, Comm. Algebra, 44(5) (2016), 2243-2255.

[7] B. Huppert, Endliche Gruppen. I, Die Grundlehren der Mathematischen Wissenschaften, Band 134, Springer-Verlag, Berlin-New York, 1967.

[8] G. D. James, The irreducible representations of the symmetric groups, Bull. London Math. Soc., 8(3) (1976), 229-232.

[9] G. D. James, The Representation Theory of the Symmetric Groups, Lecture Notes in Mathematics, 682, Springer, Berlin, 1978.

[10] G. D. James, Representations of General Linear Groups, London Mathematical Society Lecture Note Series, 94, Cambridge University Press, Cambridge, 1984. 
[11] J. Müller and J. Orlob, On the structure of the tensor square of the natural module of the symmetric group, Algebra Colloq., 18(4) (2011), 589-610.

[12] W. Plesken, Beiträge zur Bestimmung der endlichen irreduziblen Untergruppen von GL(n,Z) und ihrer ganzzahligen Darstellungen. PhD thesis, RWTH Aachen, 1974.

[13] W. Plesken, On absolutely irreducible representations of orders, In Hans Zassenhaus, editor, Number theory and algebra, Academic Press, New York, (1977), 241-262.

[14] W. Plesken, Gruppenringe über lokalen Dedekindbereichen, Habilitation, RWTH Aachen, 1980.

[15] W. Plesken, Group Rings of Finite Groups over p-adic Integers, Lecture Notes in Mathematics, 1026, Springer-Verlag, Berlin, 1983.

[16] L. Solomon, Zeta functions and integral representation theory, Advances in Math., 26(3) (1977), 306-326.

Susanne Danz (Corresponding Author)

Department of Mathematics and Geography

KU Eichstätt-Ingolstadt

Ostenstr. 26

85072 Eichstätt

Germany

e-mail: susanne.danz@ku.de

\section{Tommy Hofmann}

Department of Mathematics

University of Kaiserslautern

P.O. Box 3049

67653 Kaiserslautern

Germany

e-mail: thofmann@mathematik.uni-kl.de 